\title{
Analysis of Entropy Generation in Biomimetic Electroosmotic Nanofluid Pumping Through a Curved Channel with Joule Dissipation
}

\author{
V. K. Narla ${ }^{\mathrm{a}}$, Dharmendra Tripathi ${ }^{\mathrm{b}, *}$, O. Anwar Bégc \\ ${ }^{a}$ Department of Mathematics, GITAM University, Hyderabad-502329, India \\ ${ }^{b}$ Department of Sciences and Humanities, National Institute of Technology, \\ Uttarakhand-246174, India \\ ${ }^{c}$ Aeronautical and Mechanical Engineering, University of Salford, Manchester, \\ $M 54 W T, U K$
}

\begin{abstract}
Biomimetic designs are increasingly filtering into new areas of technology in recent years. Such systems exploit characteristics intrinsic to nature to achieve enhanced adaptivity and efficiency in engineering applications. Peristaltic propulsion is an example of such characteristics and in the current article it is explored as a feasible mechanism for deployment in electrokinetic pumping of nanofluids through a curved distensible conduit as a model for a bioinspired smart device. The unsteady mass, momentum, energy and nanoparticle concentration conservation equations for a Newtonian aqueous ionic fluid under an axial electrical field are formulated and simplified using lubrication approximations and low zeta potential (Debye Hückel linearization). A dilute nanofluid is assumed with Brownian motion and thermophoretic body forces present. The reduced non-dimensional conservation equations are solved with the symbolic software, Mathematica 9 via the NDSolve algorithm for velocity, temperature, nano-particle concentration distributions for low zeta potential. An entropy generation analysis is also conducted. The influence of curvature parameter, maximum electroosmotic velocity (Helmholtz-Smoluchowski velocity), inverse EDL thickness parameter, zeta potential ratio and Joule heating parameter on transport characteristics
\end{abstract}

\footnotetext{
*Corresponding author

Email addresses: vknarla@gmail.com (V. K. Narla), dtripathi@nituk.ac.in (Dharmendra Tripathi), gortoab@gmail.com (O. Anwar Bég)
} 
is evaluated with the aid of graphs and contour plots. Temperature profiles are elevated with positive Joule heating and reduced with negative Joule heating whereas the opposite behaviour is observed for the nano-particle concentrations.

Keywords: Peristaltic biomimetic pumping, aqueous ionic nanofluid, electroosmosis, Joule heating, curvature, entropy generation analysis

\section{Introduction}

Bio-inspired engineering systems embrace established and self-adaptive features observed in natural systems. In the 21st century with ever-increasing emphasis on optimization of technologies, numerous biomimetic technologies are being developed. These have utilized a diverse array of natural mechanisms and geometries and have penetrated practically all aspects of engineering including ion transport behavior mimicked in a biological protein-based ion pump [1], biomimetic DNA-based channels [2], lipid-membrane inspired bioconvectionnano fuel cells [3], nanowire membranes featuring biological wettability [4], tree-inspired transpiration cooling systems [5], pectoral fininspired wave energy conversion devices [6], photosynthetic-based fuel systems [7], fish-based micro-hydronautics (naval propulsion) [8, 9] and superhydrophobic and hydrophilic aircraft micro/nanopatterned coatings inspired by leaves [10]. These examples have shown considerable improvement in engineering performance and have led to more sustainable, durable and "smart" systems. Among the numerous mechanisms encountered in nature, peristalsis is particularly attractive to the engineer owing to the minimization of working parts and the resilience achieved in practical implementation. Peristalsis is a rhythmic contraction/expansion of muscles which arises in many biological processes. A seminal study of the fluid dynamics of peristalsis was presented by Jaffrin and Shapiro [11] who popularized the lubrication and long-wave approximations and established the theoretical foundation for many future investigations including reflux, trapping, retrograde and other phenomena. In recent years peristaltic pumping mechanisms have been deployed in many intriguing directions including biomimetic displacement pumps [12], antagonistic actuators in soft robotic locomotion [13], electro-pneumatics [14], peristaltic control of soft-bodied swallowing robots [15], Peristaltic electrohydrodynamic control of plasma actuators in supersonic aerodynamics and flexible silicone-based soft robotic ring actuators [16]. Mathematical mod- 
elling studies of peristaltic propulsion have also received significant attention [17, 18, 19, 20, 21, 22]. Manzoor et al. [23] obtained computational solutions for peristaltic pumping of magnetized rheological biofluids in ciliated conduits with thermal convection and permeability effects. Guo et al. [24] analyzed trapping phenomena and wave amplitude effects in hydromagnetic pumping of viscoelastic fluids in porous media-filled tubes. AbdElmaboudet al. [25] considered nonlinear viscosity variation in finite tube peristaltic pumping. Carlos Gómez-Blanco et al. [26] utilized ABAQUS finite element software to simulate peristaltic pumping in ureteral stents with Mooney-Rivlin, Yeoh, and Ogden nonlinear elastic wall models.

In recent years another significant development in engineering is the application of nanofluids. The term "nanofluid" was introduced by Choi [27], to characterize engineered colloids composed of nanoparticles dispersed in a base fluid. In comparison with milli and micro sized particle slurries explored in past, the nanoparticles are closer in molecular dimension to the particles of the base fluid. A nanofluid comprises a colloidal mixture of a small quantity of conducting nanoparticles suspended in a base fluid, such as water. Nanofluids have been shown to exhibit high, non-linear and anomalous thermal conductivity, compared to the base fluid and to achieve significant elevations in heat transfer rates. Nanofluid dynamics involves four scales: the molecular scale, the microscale, the macroscale and the megascale and an interaction is known to take place between these scales. Diverse types of nanofluids can be synthesized by combining different nano-particles (e.g. metallic oxides, silicon carbides, carbon nanotubes) with different base fluids. Nanofluids have been employed in an extensive spectrum of applications in both energy and medical engineering including thermal storage [28], direct absorber solar power collectors [29], PEM fuel cells [30], solar gel coatings [30]. Although many analytical models for nanoscale flows have been developed [31, 32, 33], a popular approach remains the Buongiorno twocomponent laminar four-equation non-homogeneous equilibrium model [34] which emphasizes the role of thermophoretic forces and Brownian motion dynamics as the key contributors to thermal conductivity enhancement. This model fits well into multi-physical fluid dynamics.

In micro-channel systems and numerous other areas of biotechnology (e.g. chemical separation), electro-osmotic flows arise. They are a special case of electro-kinetic flows and characterized by transport in which charged particles or ionic fluids (electrolytes) are mobilized by electrical fields in conduits which have charged walls. In electro-osmotic flows the electrostatic body 
force is of high magnitude in the electrical double layers. However, the proximity of the charged no-slip surface leads to substantial viscous drag. Electro-osmotic flow is therefore generated by an applied potential or electric field in a system that has an imbalance of charge (e.g. charged walls). Important biological applications of electro-osmosis include translocation in trees Polevoi [35], cell culture and tissue scaffolding devices, BioMEMS (biomicro-electromechanical systems) for pharmacodynamics, minimally invasive medical procedures, implantable neuro-probes and ocular implants and cellular microinjection [36]. Chakraborty [37] presented the first simulations for the modulation of peristaltic transport via an electro-osmotic body force. Tripathi et al. [38] presented the electro-osmotic pumping of nanofluids with heat and mass transfer in a micro-channel under peristaltic waves with Joule heating, Soret and Dufour cross-diffusion effects. Recently, Tripathi et al. [39] extended this model to consider Joule heating and buoyancy effects in electro-osmotic peristaltic pumping of water-based nanofluids with different wave forms. Noreen et al. [40] studied a theoretical model to to simulate electroosmotic transport via peristaltic motion in non-Darcy porous medium. More recently, Narla et al. [41] developed a two-dimensional model of electroosmotic modulated peristaltic transport in a channel with a phase shift between the walls. This model was extended by Narla et al. [42] to study uterine hydrodynamics of tapered geometry of the uterine cavity.

In many biological systems, heat transfer is present and energy losses are incurred which can cause disorder (entropy). The mitigation or reduction of energy losses is now a major focus in bio-inspired engineering systems. Avoiding or controlling this energy loss in thermal processes has gained much interest among researchers in recent years. Analysis of entropy generation is a powerful tool enabling the minimization of energy wastage or the optimum utilization for maximizing system performance. Bejan [43] pioneered the entropy generation minimization (EGM) approach, initially for industrial thermal systems. Since generically thermal processes are inherently irreversible, this leads to continuous entropy generation, which eliminates the exergy (useful energy or available energy for work) of a system via different modes of heat transfer (thermal conduction, convection and radiation). In biological systems other phenomena may also contribute to the entropy production including electrical fields (due to ions), magnetic fields (associated with iron in the haemoglobin molecule), viscosity (fluid friction), magnetic field, thermal buoyancy, species buoyancy etc. Minimization in the loss of exergy in any system is desirable since it permits optimal usage of the energy situation 
with minimum irreversibilities. This optimum condition can be assessed via entropy generation minimization (EGM). An extensive range of biological flows have been studied with this technique which utilizes the second law of thermodynamics to enable a more refined appraisal of heat transfer and mitigation of losses. Akbar et al. [44] investigated analytically the entropy generation in pumping of carbon nanotube (CNT)-nanofluids through a ciliated permeable medium, noting that entropy generation rate is enhanced with both Darcy (permeability parameter) and Brinkman number (ratio of direct heat conduction from the wall surface to the viscous heat generated by shear in the boundary layer). They also showed that Bejan number (ratio of heat transfer irreversibility to total irreversibility due to heat transfer and fluid friction) is enhanced with higher values of Brinkman and Darcy numbers. Escandón et al. [45] developed asymptotic solutions for entropy generation in electroosmotic non-Newtonian flow in a microchannel, noting that the entropy generation is dominated by Joule heating. Zhao and Liu [46] investigated entropy generation in electro-osmotic flow in two-dimensional open-end and closed-end micro-channels, computing the contributions from heat conduction, viscous dissipation and Joule heating. They showed that volumetric entropy generation rates due to Joule heating is maximized at the center of the micro-channel, whereas those associated heat conduction and viscous dissipation peak in the vicinity of the micro-channel walls. They further noted that Joule heating results in an elevation with applied electrical field in both the heat conduction entropy generation number and Joule heating entropy generation number. Their simulations also indicated that for the case where temperature increment due to Joule heating exceeds the temperature difference between the inlet and the top wall, the electro-osmotic flow entropy generation due to Joule heating constitutes the major portion of the total entropy.

The above studies have generally been confined to straight channels or tubes i.e. the influence of curvature has been neglected. Several investigators have considered peristaltic pumping in curved vessels. There have been a couple of investigations of fluid transport in a curved channel undergoing peristalsis with prescribed wall motions [47, 48, 49, 50, 51, 52, 53]. Narla et al. [54] studied entropy generation analysis for peristalsis of viscous fluid in a curved geometry. They reported that curvature of channel is one of the strong sources of entropy generation. In another study, Narla et al. [55] investigated unsteady magnetized non-Newtonian peristaltic pumping in distensible curved conduits. Very recently, Narla and Tripathi [56] per- 
formed electro-osmotic fluid dynamic analysis to study the coupling force of electrokinetic force and wall motility in a two-dimensional curved channel undergoing peristalsis to reflect transient blood flow in curved microvessels.

In the current article, a mathematical model is developed for the propulsion of electro-osmotic ionic nanofluids in a curved deformable microchannel under peristaltic waves. Joule heating and thermal and species buoyancy effects are included. The transformed conservation equations are simplified for the case of low zeta potential (Debye Hckel linearization) and solved analytically. With the aid of Mathematica 9 symbolic software solutions are evaluated numerically. Entropy generation minimization analysis is also included, and expressions derived for entropy generation rate and Bejan number. The impact of curvature parameter, maximum electroosmotic velocity (Helmholtz-Smoluchowski velocity), inverse EDL thickness parameter, zeta potential ratio and Joule heating parameter on velocity, temperature, nanoparticle concentration, streamline, isotherm and iso-concentration characteristics are examined, for fixed values of the buoyancy, geometric and nanoscale parameters. The study is motivated by providing a deeper insight thermodynamic optimization of bio-inspired electro-osmotic nanofluid micro-channel pumping systems of relevance in bionics and nanofluidic devices.

\section{Mathematical model}

The physical model of the "smart" biomimetic electro-nanofluidic micropump is shown in Fig. 1. It comprises a distensible curved micro-channel with width $2 a$. The channel has center $O$ and radius of curvature $R$. A curvilinear coordinate system $(x, r)$ in the laboratory frame is adopted with a velocity vector $\bar{V}=u(x, r, t) \widehat{e}_{x}+v(x, r, t) \widehat{e}_{r}$. The peristaltic propulsion is mobilized via both the electrokinetic force and the transverse deflections of sinusoidal waves of small amplitude $b$ that are imposed on the deformable (flexible) walls of the micro-channel. No-slip, isothermal $\left(T=T_{0}\right)$ and isosolutal $\left(C=C_{0}\right)$ conditions are enforced at the walls of the micro-channel. The nano-particles are assumed to be spherical and of the same size and in a state of local thermal equilibrium and the ionic aqueous nanofluid is dilute. An electrical field, E, is applied to the micro-channel walls. The fluid (e.g. blood) is Newtonian and incompressible. The deformation of the micro-channel walls is simulated with the following relations:

$$
r= \pm h(x, t)= \pm\left(a-b \cos ^{2} \pi\left(\frac{x-c t}{\lambda}\right)\right)
$$




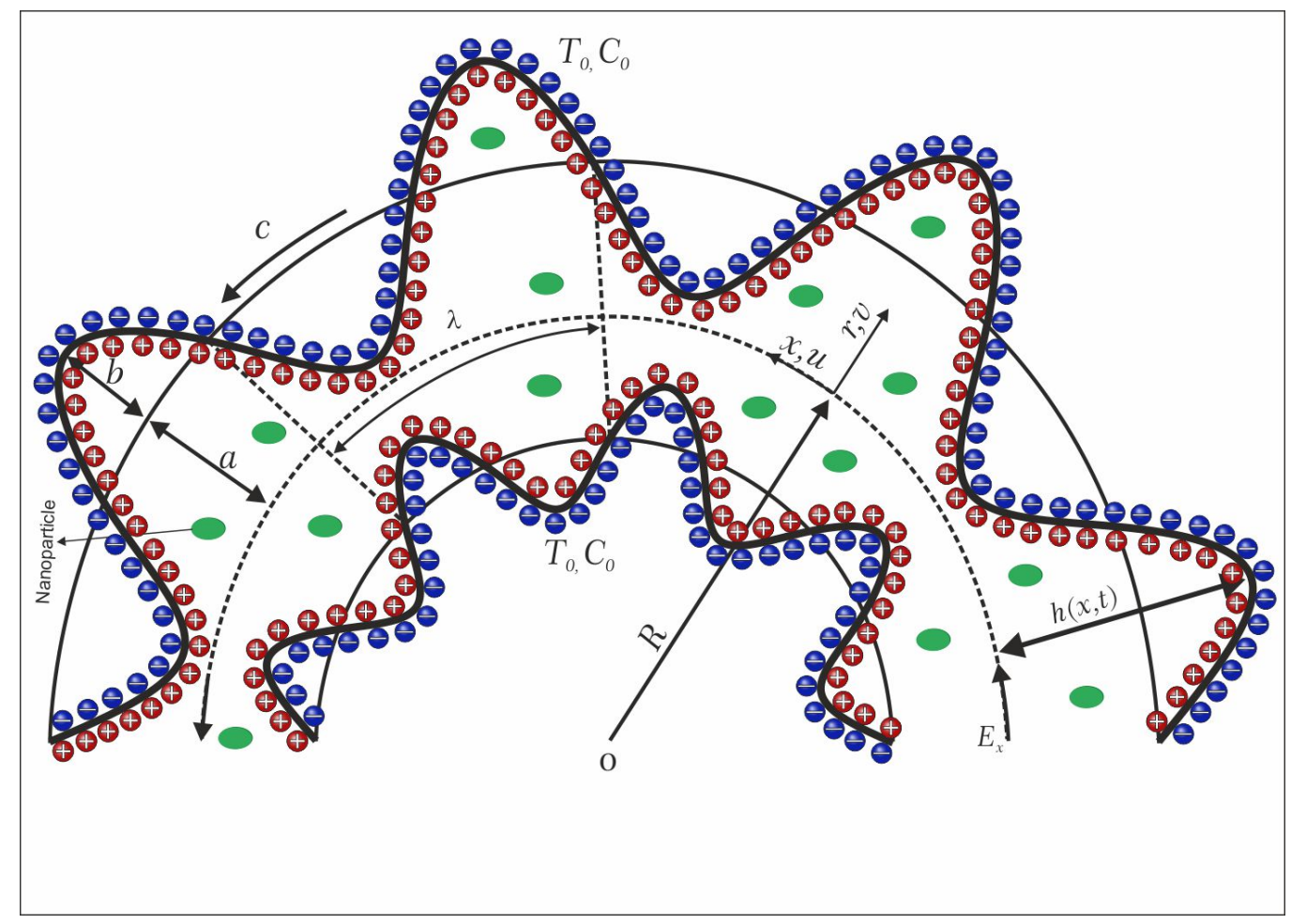

Figure 1: Schematic view of the elecroosmotic flow with peristalsis in a curved channel.

Here $r$ denotes the radial coordinate, $x$ is the axial distance, $a$ is the radius of the stationary curved channel, $b$ is the wave amplitude, $\lambda$ is the wave length, $t$ is the time, $T$ is the wave period, and $h$ is the radial displacement of the wave from the channel central line. The wavelength is considered to be much greater than the micro-channel width $(a / \lambda<<1)$.

\subsection{Electro-osmotic flow analysis}

According to electrostatics theory, the electric potential, $\Phi$, is governed by well-known Poisson equation:

$$
\nabla^{2} \Phi=-\frac{\rho_{e}}{\varepsilon} .
$$

In general, the ion distribution is affected by two different contributions: $(a)$ the externally applied potential, $\tilde{\Phi}$, and $(b)$ the electrical double layer (EDL) 
potential, $\Psi$, associated surface zeta-potential, $\zeta$. However, it is important to mention that, while the externally applied potential is presented by Laplace equation, the equation (2) with net charge density in a unit volume of a symmetric electrolyte, i.e. $n_{+0}=n_{-0}=n_{0}, z_{+}=z_{-}=z$, can be decomposed as

$$
\nabla^{2} \tilde{\Phi}=0, \quad \text { and } \quad \nabla^{2} \Psi=-\frac{z e}{\varepsilon}\left(n_{+}-n_{-}\right) .
$$

where $n_{0}=C N_{A}$ ( $C$ is the molar concentration of ions and $N_{A}$ is Avogadros number), is the bulk concentration of ions and $z$ is the valence of the ions. In order to define potential distribution, it is important to analyze species conservation equation. The Nernst-Planck equation for the curved geometry [56], in the absence of chemical reactions, can be expressed as

$$
\begin{aligned}
& \left(\frac{\partial n_{ \pm}}{\partial t}+u \frac{R}{R+r} \frac{\partial n_{ \pm}}{\partial x}+v \frac{\partial n_{ \pm}}{\partial r}\right)=D_{ \pm}\left(\nabla^{2} n_{ \pm}\right) \\
& \quad \pm \frac{D_{ \pm} z_{ \pm} e}{k_{B} T_{1}}\left[\frac{R}{R+r} \frac{\partial}{\partial x}\left\{n_{ \pm} \frac{R}{R+r} \frac{\partial \Psi}{\partial x}\right\}+\frac{1}{R+r} \frac{\partial}{\partial r}\left\{(R+r) n_{ \pm} \frac{\partial \Psi}{\partial r}\right\}\right]
\end{aligned}
$$

where $D$ is the diffusivity of an ionic species, $k_{B}$ is the Boltzmann constant, and $T_{1}$ absolute temperature.

\subsection{Conservation equations for electro-thermo-nanofluidic transport}

The negatively charged wall of the micro-channel attracts opposite ions in the ionic nanofluid generating a layer of positively charged fluid near the wall and at the same time repels the counter-ions. The thin layer of immobile counter-ions covering the inner side of the wall is known as the Stern layer. Below the Stern layer, a thicker layer of moving counter-ions develops. The combination of the Stern layer and the thicker layer is known as the electric double layer (EDL). The electro-kinetic body force emerges by connecting the system to an external applied electric field. The time-dependent equations for the conservation of mass, momentum, energy and nanoparticle concentration for ionic aqueous Newtonian biofluid in the curved microchannel can be written as $([47,51,56])$ :

Equation of mass conservation

$$
\frac{R}{r+R} \frac{\partial u}{\partial x}+\frac{\partial v}{\partial r}+\frac{v}{r+R}=0
$$


Equation of momentum conservation

$$
\begin{gathered}
\rho_{f}\left[\frac{\partial u}{\partial t}+(\bar{V} \cdot \nabla) u+\frac{u v}{r+R}\right]=-\frac{R}{r+R} \frac{\partial p}{\partial x}+\mu\left[\nabla^{2} u-\frac{u}{(r+R)^{2}}+\frac{2 R}{(r+R)^{2}} \frac{\partial v}{\partial x}\right] \\
+\left(1-C_{0}\right) \rho_{p} g \alpha\left(T-T_{0}\right)+\left(\rho_{p}-\rho_{f}\right) g \beta\left(C-C_{0}\right)+\rho_{e} E_{x}, \\
\rho_{f}\left[\frac{\partial v}{\partial t}+(\bar{V} \cdot \nabla) v-\frac{u^{2}}{r+R}\right]=-\frac{\partial p}{\partial r}+\mu\left[\nabla^{2} v-\frac{v}{(r+R)^{2}}-\frac{2 R}{(r+R)^{2}} \frac{\partial u}{\partial x}\right]+\rho_{e} E_{r},
\end{gathered}
$$

Equation of energy conservation

$$
\begin{aligned}
{\left[\frac{\partial T}{\partial t}+(\bar{V} \cdot \nabla) T\right] } & =\frac{K}{(\rho c)_{f}} \nabla^{2} T+\tau\left[D_{B}(\nabla C \cdot \nabla T)+\frac{D_{T}}{T_{m}}(\nabla T \cdot \nabla T)\right] \\
& +\frac{\mu}{(\rho c)_{f}}\left[2\left\{\left(\frac{\partial v}{\partial r}\right)^{2}+\left(\frac{R}{r+R} \frac{\partial u}{\partial x}+\frac{v}{r+R}\right)^{2}\right\}\right. \\
& \left.+\left(\frac{\partial u}{\partial r}+\frac{R}{r+R} \frac{\partial v}{\partial x}-\frac{u}{r+R}\right)^{2}\right]+\frac{\sigma_{e}}{(\rho c)_{f}} E_{x}^{2}
\end{aligned}
$$

Equation of nanoparticle concentration (species)

$$
\left[\frac{\partial C}{\partial t}+(\bar{V} \cdot \nabla) C\right]=D_{B} \nabla^{2} C+\frac{D_{T}}{T_{m}} \nabla^{2} T
$$

Here the vector operators $\bar{V} \cdot \nabla$ and $\nabla^{2}$ are given in the Appendix Appendix A. The parameters $p, \rho, \mu, K, c_{p}, T, C, D_{B}, D_{T}, \tau=\frac{\rho_{c p}}{\rho_{c f}}, \alpha, \beta, g, \sigma_{e}$ and $E$ designate respectively the pressure, density, dynamic viscosity, thermal conductivity, specific heat at constant pressure, temperature, nano-particle concentration, Brownian diffusion coefficient, thermophoretic diffusion coefficient, ratio of the effective heat capacity of the nanoparticle material and heat capacity of the fluid, coefficient of linear thermal expansion of the fluid, coefficient of expansion with concentration, acceleration due to gravity, electrical conductivity, and applied electrical field. The electrokinetic body force $\mathbf{F}_{E}$ is here given as $\mathbf{F}_{E}=\rho_{e} \mathbf{E}=\rho_{e}\left(E_{x} \widehat{e}_{x}+E_{r} \widehat{e}_{r}\right)$, where $\mathbf{E}$ the applied external electric field and $\rho_{e} \equiv e\left(z_{+} n_{+}-z_{-} n_{-}\right)$is the net charge density of the aqueous medium of permittivity $\varepsilon$ and $e$ is the elementary electronic charge $\left(1.6 \times 10^{-19} C\right)$, here $z_{ \pm}$and $n_{ \pm}$are the valence and number densities of coions and counterions respectively. The body force induces a bulk fluid motion generally referred to as electro-osmotic flow and the relationship between the 
electroosmotic flow and the applied electric field $\mathbf{E}$ in curved micro-channels is given by:

$$
\mathbf{E}=-\frac{R}{(R+r)} \frac{\partial \Phi}{\partial x} \widehat{e}_{x}-\frac{\partial \Phi}{\partial r} \widehat{e}_{r},
$$

where $\Phi$ is the electric potential.

\subsection{Non-dimensional analysis}

The flow governing equations can be solved by applying lubrication approximation. It is assumed that Reynolds number $(R e)$ and the local slope of the channel wall are very small, which implies negligible inertial effects in the micro-channel $(\lambda / a \gg 1)$. It is judicious to introduce non-dimensional variables, as follows:

$$
\begin{aligned}
& x^{\prime}=\frac{x}{\lambda}, r^{\prime}=\frac{r}{a}, u^{\prime}=\frac{u}{c}, v^{\prime}=\frac{v}{\delta c}, \delta=\frac{a}{\lambda}, t^{\prime}=\frac{c t}{\lambda} h^{\prime}=\frac{h}{a}, \phi=\frac{b}{a}, \kappa=\frac{R}{a}, \\
& L^{\prime}=\frac{L}{\lambda}, p^{\prime}=\frac{a^{2} p}{\mu c \lambda}, R e=\frac{\rho_{f} c a}{\mu}, \psi^{\prime}=\frac{\psi}{a c}, F=\frac{Q}{a c}, \quad \sigma=\frac{C-C_{0}}{C_{0}}, \\
& \theta=\frac{T-T_{0}}{T_{0}}, \operatorname{Pr}=\frac{\mu c_{p}}{K}, E c=\frac{c^{2}}{c_{p} T_{0}}, N b=\frac{\tau D_{B} C_{0}}{\nu}, N t=\frac{\tau D_{T} T_{0}}{T_{m} \nu}, \\
& G r=\frac{\alpha g T_{0} a^{2}\left(1-C_{0}\right)}{c \nu}, G c=\frac{\left(\rho_{p}-\rho_{f}\right) \beta g C_{0} a^{2}}{c \mu}, \Psi^{\prime}=\frac{\Psi}{\Psi_{0}}, \zeta^{\prime}=\frac{\zeta}{\Psi_{0}}, \\
& U_{H S}^{\prime}=\frac{U_{H S}}{c}, P e=\frac{c \lambda}{D}, S=\frac{\sigma_{e} E_{x}^{2} a^{2}}{K T_{0}}, n^{\prime}=\frac{n}{n_{0}}, m^{\prime}=m a .
\end{aligned}
$$

Here $x^{\prime}$ is dimensionless axial coordinate, $r^{\prime}$ is dimensionless radial coordinate, $u^{\prime}$ is dimensionless axial velocity, $v^{\prime}$ is dimensionless transverse (radial) velocity, $\delta$ is the wave number, $t^{\prime}$ is dimensionless time, $h^{\prime}$ is dimensionless wall deformation, $\phi$ is the amplitude ratio or the occlusion parameter, $\kappa$ is the curvature parameter (larger values imply straighter microchannels and smaller values correspond to more curved geometries), $L^{\prime}$ is the dimensionless axial length of the finite micro-channel, $p^{\prime}$ is dimensionless pressure, $R e$ is the Reynolds number (based on the micro-channel semiwidth as the scaling dimension), $F$ is the volumetric flow rate, $\sigma$ is the non-dimensional nano-particle concentration, $\theta$ is the non-dimensional temperature, $\operatorname{Pr}$ is the Prandtl number, $E c$ is the Eckert number, $N b$ is the Brownian motion parameter, $N t$ is the thermophoresis parameter, $G r$ is the thermal buoyancy parameter i.e. local temperature Grashof number, $G c$ is 
the concentration buoyancy parameter i.e. local nanoparticle Grashof number, $\Psi^{\prime}$ is the dimensionless thermal potential (in which $\Psi_{0}=k_{B} T_{0} / e z$ is the thermal potential), $\zeta^{\prime}$ is dimensionless surface zeta-potential, $U_{H S}^{\prime}$ is nondimensional Helmholtz-Smoluchowski velocity (where $U_{H S}=-\left(\epsilon_{e} \Psi_{0} E_{x}\right) / \mu$ is the Helmholtz-Smoluchowski velocity i.e. maximum electro-osmotic velocity), and $P e$ is ionic Péclet number (ratio of the contributions to ionic mass transport by convection to those by diffusion), $S$ Joule heating parameter, $n^{\prime}$ is dimensionless bulk concentration of ions, and $m^{\prime}$ is the Debye-Hückel parameter.

The non-dimensional form of the Nernst-Planck equation (4) is written as (after omitting all the primes):

$$
\begin{gathered}
P e \delta^{2}\left[u \frac{\kappa}{\kappa+r} \frac{\partial n_{ \pm}}{\partial x}+v \frac{\partial n_{ \pm}}{\partial r}\right]=\left(\delta^{2}\left(\frac{\kappa}{r+\kappa}\right)^{2} \frac{\partial^{2} n_{ \pm}}{\partial x^{2}}+\frac{\partial^{2} n_{ \pm}}{\partial r^{2}}+\frac{1}{r+\kappa} \frac{\partial n_{ \pm}}{\partial r}\right) \\
\pm\left(\delta^{2} \frac{\kappa}{\kappa+r} \frac{\partial}{\partial x}\left\{n_{ \pm} \frac{\kappa}{\kappa+r} \frac{\partial \Psi}{\partial x}\right\}+\frac{1}{\kappa+r} \frac{\partial}{\partial r}\left\{(\kappa+r) n_{ \pm} \frac{\partial \Psi}{\partial r}\right\}\right),
\end{gathered}
$$

the magnitude of the Péclet number $P e$ may range from $\sim 0.01-1$ for the typical values assumed as $c \sim 10^{-2} \mathrm{~m} / \mathrm{s}, \lambda \sim 10 \mu \mathrm{m}$, and $D \sim 10^{-9} \mathrm{~m}^{2} / \mathrm{s}$. The non-linear terms in the equation (12) are of $O\left(P e \delta^{2}\right)$. Therefore, for $\delta \rightarrow 0$ and $P e \delta^{2} \rightarrow 0$, the reduced form of the Nernst-Planck equation can be written as:

$$
\frac{\partial^{2} n_{ \pm}}{\partial r^{2}}+\frac{1}{r+\kappa} \frac{\partial n_{ \pm}}{\partial r}=\mp \frac{1}{\kappa+r} \frac{\partial}{\partial r}\left\{(\kappa+r) n_{ \pm} \frac{\partial \Psi}{\partial r}\right\}
$$

this is subject to bulk conditions:

$$
n_{ \pm}(\Psi=0)=1 \text { and } \frac{\partial n_{ \pm}}{\partial r}=0 \text { where } \frac{\partial \Psi}{\partial r}=0 .
$$

The solution of equation (13) with equation (14) gives the Boltzmann distributions for the ions as:

$$
n_{ \pm}=\exp (\mp \Psi) .
$$

Combining equations (3) and (15), we obtain the well-known nonlinear PoissonBoltzmann equation (non-dimensional form), that gives electric potential 
distribution in the EDL, i.e,

$$
\delta^{2}\left(\frac{\kappa}{r+\kappa}\right)^{2} \frac{\partial^{2} \Psi}{\partial x^{2}}+\frac{1}{r+\kappa} \frac{\partial}{\partial r}\left\{(r+\kappa) \frac{\partial \Psi}{\partial r}\right\}=m^{2} \sinh (\Psi),
$$

Invoking the classical lubrication theory framework $\left(\delta^{2} R e \rightarrow 0, \delta^{2} \rightarrow 0\right)$, the momentum equations (6) and (7) after eliminating the pressure term, the energy equation (8), the nanoparticles concentration equation (9), and the nonlinear Poisson-Boltzmann equation (16) emerges in terms of the above dimensionless parameters and stream function $u=\frac{\partial \psi}{\partial r}, v=-\frac{\kappa}{r+\kappa} \frac{\partial \psi}{\partial x}$, as follows, after omitting the primes:

$$
\begin{gathered}
\frac{\partial}{\partial r}\left[\frac{1}{r+\kappa} \frac{\partial}{\partial r}\left\{(r+\kappa)^{2}\left(\frac{\partial^{2} \psi}{\partial r^{2}}-\frac{1}{r+\kappa} \frac{\partial \psi}{\partial r}\right)\right\}+(r+\kappa)(G r \theta+G c \sigma)\right] \\
=-U_{H S} \frac{\partial^{2}}{\partial r^{2}}\left\{(r+\kappa) \frac{\partial \Psi}{\partial r}\right\} \\
\frac{1}{P r}\left(\frac{\partial^{2} \theta}{\partial r^{2}}+\frac{1}{r+\kappa} \frac{\partial \theta}{\partial r}\right)+E c\left(\frac{\partial^{2} \psi}{\partial r^{2}}-\frac{1}{r+\kappa} \frac{\partial \psi}{\partial r}\right)^{2}+N b\left(\frac{\partial \theta}{\partial r} \frac{\partial \sigma}{\partial r}\right)+N t\left(\frac{\partial \theta}{\partial r}\right)^{2}+S=0 \\
\frac{\partial^{2} \sigma}{\partial r^{2}}+\frac{1}{r+\kappa} \frac{\partial \sigma}{\partial r}+\frac{N t}{N b}\left(\frac{\partial^{2} \theta}{\partial r^{2}}+\frac{1}{r+\kappa} \frac{\partial \theta}{\partial r}\right)=0 \\
\frac{1}{r+\kappa} \frac{\partial}{\partial r}\left\{(r+\kappa) \frac{\partial \Psi}{\partial r}\right\}=m^{2} \sinh (\Psi)
\end{gathered}
$$

The corresponding dimensionless boundary conditions are given by

$\psi( \pm h)= \pm \frac{F}{2}, \frac{\partial \psi}{\partial r}( \pm h)=0, \theta( \pm h)=0, \sigma( \pm h)=0, \Psi(-h)=\zeta_{1}, \Psi(h)=\zeta_{2}$.

The non-dimensional wall deformation equation take the form:

$$
h=1-\phi \cos ^{2} \pi(x-t) .
$$

\subsection{Analytical solution for low zeta potentials}

The electric potential and ion distributions are de-coupled from the NavierStokes equations (i.e., independent of fluid velocity). When using the DebyeHückel approximation, equation (20) can be linearized by virtue of the approximation, $\sinh (x) \sim x$. This approximation is valid for many applications, when an aqueous solution $\mathrm{pH}$ value is near neutral, and the magnitude 
of zeta potentials $\left|\zeta_{1}\right|$ and $\left|\zeta_{2}\right|$ are $25 \mathrm{mV}$ or less. Therefore, an analytical solution can be derived for the Poisson-Boltzmann equation. The simplified dimensionless Poisson-Boltzmann equation with corresponding boundary conditions are

$$
\frac{1}{r+\kappa} \frac{\partial}{\partial r}\left[(r+\kappa) \frac{\partial \Psi}{\partial r}\right]-m^{2} \Psi=0, \quad \Psi(-h)=\zeta_{1}, \Psi(h)=\zeta_{2} .
$$

The analytical solution of the boundary value problem in Eq. (23) can be obtained as

$$
\Psi(x, r)=\zeta_{1}\left[\frac{K_{2}-R_{\zeta} K_{1}}{I_{1} K_{2}-I_{2} K_{1}} I_{0}[m(r+\kappa)]+\frac{R_{\zeta} I_{1}-I_{2}}{I_{1} K_{2}-I_{2} K_{1}} K_{0}[m(r+\kappa)]\right] .
$$

Here, $I_{0}$ and $K_{0}$ are modified Bessel functions of first and second kind of order 0 respectively, $I_{1}=I_{0}[m(k-h)], I_{2}=I_{0}[m(k+h)], K_{1}=K_{0}[m(k-h)]$ and $K_{2}=K_{0}[m(k+h)]$.

\subsection{Entropy Analysis}

Entropy generation is closely associated with thermodynamic irreversibility, which is encountered in all practical heat transfer processes. Different sources are responsible for entropy generation such as heat transfer in the presence of temperature difference, the mass transfer in the presence of species concentration difference, the viscous dissipation and body forces (e.g. electrical, magnetic etc). The volumetric rate of entropy generation is defined as:

$$
E_{G}=\frac{K}{T_{1}^{2}}(\nabla T)^{2}+\frac{\mu}{T_{1}} \bar{\Sigma}+\frac{R D}{C_{1}}(\nabla C)^{2}+\frac{R D}{T_{1}}(\nabla T \cdot \nabla C)+\frac{\sigma_{e}}{T_{1}} E_{x}^{2},
$$

here $\bar{\Sigma}$ is viscous dissipation, $T_{1}$ and $C_{1}$ are respectively the reference temperature and concentration. The volumetric rate of local entropy generation for incompressible Newtonian electro-osmotic fluid in a curved channel takes 
the following form:

$$
\begin{aligned}
E_{G} & =\frac{K}{T_{1}^{2}}\left[\left(\frac{R}{r+R} \frac{\partial T}{\partial x}\right)^{2}+\left(\frac{\partial T}{\partial r}\right)^{2}\right]+\frac{R D}{C_{1}}\left[\left(\frac{R}{r+R} \frac{\partial C}{\partial x}\right)^{2}+\left(\frac{\partial C}{\partial r}\right)^{2}\right] \\
& +\frac{\mu}{T_{1}}\left[2\left\{\left(\frac{\partial v}{\partial r}\right)^{2}+\left(\frac{R}{r+R} \frac{\partial u}{\partial x}+\frac{v}{r+R}\right)^{2}\right\}+\left(\frac{\partial u}{\partial r}+\frac{R}{r+R} \frac{\partial v}{\partial x}-\frac{u}{r+R}\right)^{2}\right] \\
& +\frac{R D}{T_{1}}\left[\left(\frac{R}{r+R}\right)^{2} \frac{\partial T}{\partial x} \frac{\partial C}{\partial x}+\frac{\partial T}{\partial r} \frac{\partial C}{\partial r}\right]+\frac{\sigma_{e}}{T_{1}} E_{x}^{2},
\end{aligned}
$$

where $T_{0}$ is the absolute reference temperature. The dimensionless form of entropy generation rate is the entropy generation number $\left(E_{S}\right)$ which is equal to the ratio of the actual entropy generation $\left(E_{G}\right)$ to the characteristic entropy generation rate $\left(E_{C}\right)$ and is defined as

$$
E_{S}=\frac{E_{G}}{E_{C}}=E_{G} \frac{a^{2} T_{1}^{2}}{K T_{0}^{2}}
$$

Invoking the non-dimensional quantities defined in equation (11), then the equation (26) can be written as:

$$
\begin{aligned}
E_{S} & =\delta^{2}\left(\frac{k}{r+k} \frac{\partial \theta}{\partial x}\right)^{2}+\left(\frac{\partial \theta}{\partial r}\right)^{2}+\frac{E c P r}{\Omega}\left[2 \delta^{2}\left\{\left(\frac{k}{r+k} \frac{\partial u}{\partial x}+\frac{v}{r+k}\right)^{2}+\left(\frac{\partial v}{\partial r}\right)^{2}\right\}\right. \\
& \left.+\left(\frac{\partial u}{\partial r}+\delta^{2} \frac{k}{r+k} \frac{\partial v}{\partial x}-\frac{u}{r+k}\right)^{2}\right]+\Gamma\left(\frac{\Lambda}{\Omega}\right)^{2}\left[\delta^{2}\left(\frac{k}{r+k} \frac{\partial \sigma}{\partial x}\right)^{2}+\left(\frac{\partial \sigma}{\partial r}\right)^{2}\right] \\
& +\Upsilon\left[\delta^{2}\left(\frac{k}{r+k}\right) \frac{\partial \theta}{\partial x} \frac{\partial \sigma}{\partial x}+\frac{\partial \theta}{\partial r} \frac{\partial \sigma}{\partial r}\right]+\frac{S}{\Omega} .
\end{aligned}
$$

Here, the dimensionless parameters are $\Gamma=\frac{R D C_{1}}{K}, \Upsilon=\frac{R D T_{1} C_{0}}{K T_{0}}, \Omega=\frac{T_{0}}{T_{1}}$ and $\Lambda=\frac{C_{0}}{C_{1}}$. The average entropy generation rate (over a cross-section), $E_{S, A v g}$. can be defined as follows:

$$
E_{S, A v g .}=\frac{\int_{A} E_{S} d A}{\int_{A} d A} .
$$


The entropy generation rate due to heat transfer $N_{H}$, to the total entropy generation, $N_{S}$ is called the Bejan number, Be:

$$
B e=\frac{N_{H}}{N_{S}}=\frac{\left(\frac{\partial \theta}{\partial r}\right)^{2}}{\frac{E c P r}{\Omega}\left(\frac{\partial u}{\partial r}-\frac{u}{r+k}\right)^{2}+\Gamma\left(\frac{\Lambda}{\Omega}\right)^{2}\left(\frac{\partial \sigma}{\partial r}\right)^{2}+\Upsilon \frac{\partial \theta}{\partial r} \frac{\partial \sigma}{\partial r}+\frac{S}{\Omega}} .
$$

This also quantifies the irreversibility distribution. Bejan number, $B e$ generally lies between 0 and 1 i.e, $0 \leq B e \leq 1$. Therefore, if $B e=0, N_{S}$ dominates $N_{H}$ and vice versa if $B e=1$. Furthermore when $B e=0.5$, the fluid friction contribution in entropy generation and the irreversibility due to heat transfer are equal i.e., $N_{H}=N_{S}$.

\section{Numerical results and discussion}

Selected computations have been presented for for velocity, temperature, nano-particle concentration, streamline, temperature (isotherms), nano-particle concentration (iso-solutes) contours, average entropy generation rate and Bejan number. All numerical evaluation has been executed in Mathematica 9 symbolic software via the efficient NDSolve algorithm. The results are visualized graphically for the influence of selected parameters in Figs. 2-10. Since many parameters arise in the model, only five are selected here, namely the curvature parameter $(\kappa)$, maximum electroosmotic velocity (HelmholtzSmoluchowski velocity) $\left(U_{H S}\right)$, inverse EDL thickness parameter $(m)$, zeta potential $\operatorname{ratio}\left(R_{\zeta}\right)$ and Joule heating parameter $(S)$. Default values of the other parameters are as follows: $\phi=0.6$ (intermediate wave amplitude), $\kappa=2$ (strong curvature), $Q_{T}=1.2$ (high time averaged flow rate), $U_{H S}=-1$ (axial electrical field applied in negative x-direction), $E c=1$ (strong viscous dissipation), $\operatorname{Pr}=1$ (momentum and thermal diffusivity are equal and at the walls in the hydrodynamic entry region the velocity and thermal boundary layer thicknesses are equal), $G r=G c=1$ (thermal buoyancy and nanoparticle solutal buoyancy force are both equal to the viscous hydrodynamic force), $N t=0.5$ (intermediate thermophoretic body force), $N b=0.5$ (small nano-particles and mediocre Brownian diffusion), $S=1$ (strong Joule electrical dissipation), $m=2$ (low EDL thickness), $R_{\zeta}=1$ (zeta potentials $\left|\zeta_{1}\right|$ and $\left|\zeta_{2}\right|$ are equal at the outer and inner curved walls), $x=0.3$ (axial location near the entry of the micro-channel). The maximum electro-osmotic velocity i.e. Helmholtz-Smoluchowski velocity $\left(U_{H S}\right)$ is a critical parameter 


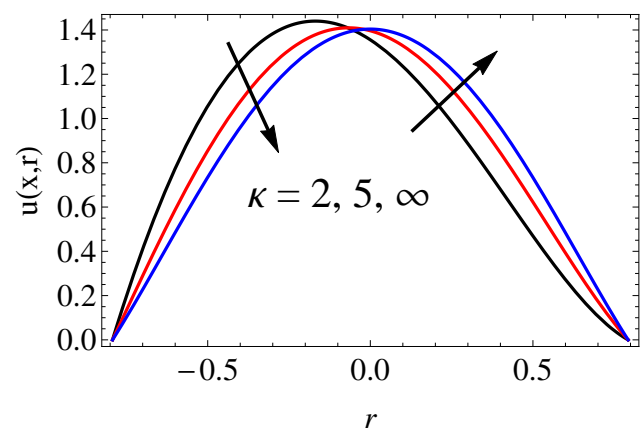

(a)

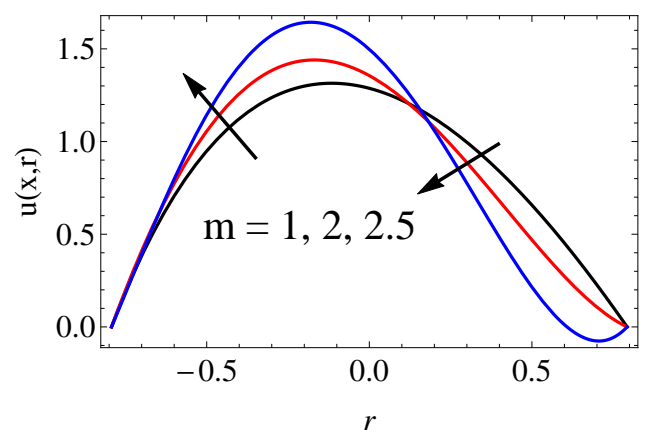

(c)

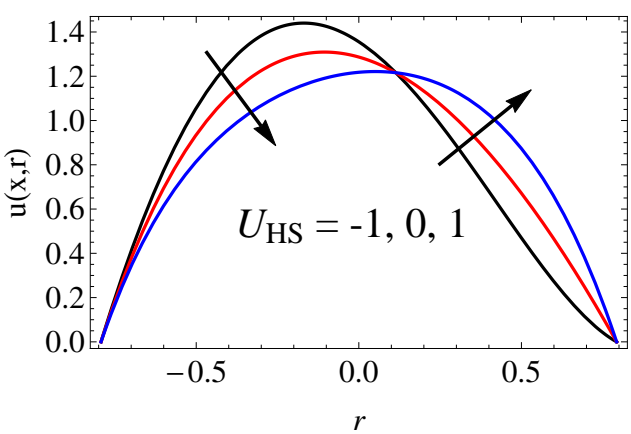

(b)

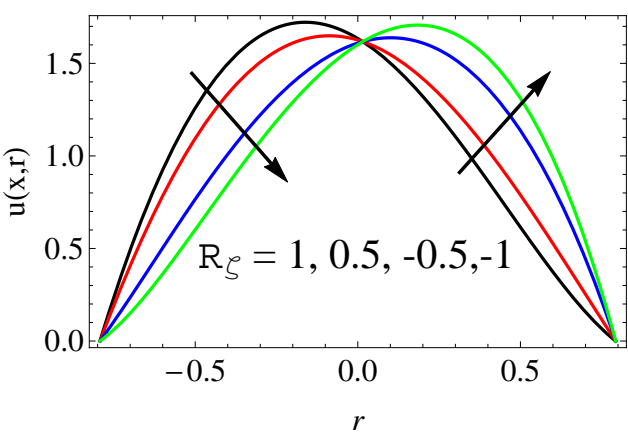

(d)

Figure 2: Velocity profiles for variation in different parameters (a) curvature parameter $\kappa$, (b) electroosmotic velocity $U_{H S}$, (c) inverse EDL thickness parameters $m$, (d) zeta potential ratios $R_{\zeta}$, with other parameters $\phi=0.6$, $\kappa=2, Q_{T}=1.5, U_{H S}=-1, E c=2, \operatorname{Pr}=1, G r=1, G c=1, N t=0.5$, $N b=0.5, S=1, m=2, R_{\zeta}=1, x=0.3, t=0$. 
of interest. This represents the influence of applied external electric field (if there is no electric field $U_{H S} \rightarrow 0$ and the model reduces to peristaltic flow of electrically non-conducting nanofluid through a curved microchannel), on velocity profile. The Joule heating parameter $(S)$ is proportional to the effect of the nano-particle volume fraction and also proportional to the diameter of nanoparticles. Nano-particle geometry effects are not explicitly considered here and have been examined by the authors and other investigators in earlier studies [55]. The case of a straight micro-channel is retrieved for $\kappa \rightarrow \infty$. The smaller the value of $\kappa$ indicates the greater curvature of the micro-channel. Figs. 2(a)-2(d) present the axial velocity plotted against radial coordinate in the micro-channel with a change in $(a)$ curvature parameter $\kappa,(b)$ Helmholtz-Smoluchowski velocity $U_{H S},(c)$ inverse EDL thickness parameter $m,(d)$ zeta potential ratio $R_{\zeta}$. With decreasing curvature parameter (stronger curved micro-channel), there is a significant increase in velocity in the lower micro-channel half space $(-1<r<0)$. However in the upper micro-channel half-space $(0<r<+1)$, this trend is reversed and the axial velocity is depleted with a reduction in curvature parameter. Evidently the location across the cross-section and the curvature exert a complex influence parameter. Nevertheless, the absolute peak axial velocity arises near the micro-channel centre line in the lower microchannel half space $(-1<r<0)$. This may be related to the assisting nature of curvature to momentum development in this vicinity. In this region the straight micro-channel scenario leads to significant deceleration. In all cases at the micro-channel walls the axial velocity vanishes in accordance with the no-slip boundary conditions imposed. Fig. 2(b) indicates that a reduction in Helmholtz-Smoluchowski velocity i.e. maximum electroosmotic velocity $U_{H S}$, induces significant acceleration in the lower microchannel half space whereas the converse behavior is generated in the upper microchannel half space. The parameter $U_{H S}=-\left(\epsilon_{e} \Psi_{0} E_{x}\right) / \mu$ is by default negative and for constant values of permittivity, applied reference electrical potential and biofluid dynamic viscosity, the axial electrical field is varied to achieve a change. In the normalized momentum conservation equation 17 the electroosmotic body force, $-U_{H S} \frac{\partial^{2}}{\partial r^{2}}\left\{(r+\kappa) \frac{\partial \Psi}{\partial r}\right\}$ is resistive for positive $U_{H S}$ and assisting for negative $U_{H S}$. Physically this corresponds to the direction of the axial electrical field. When axial electrical field is orientated in the positive x-direction, positive $E_{x}$ results in a negative $U_{H S}$ value which manifests in a positive (i.e. double negative) electro-osmotic body force which serves to 


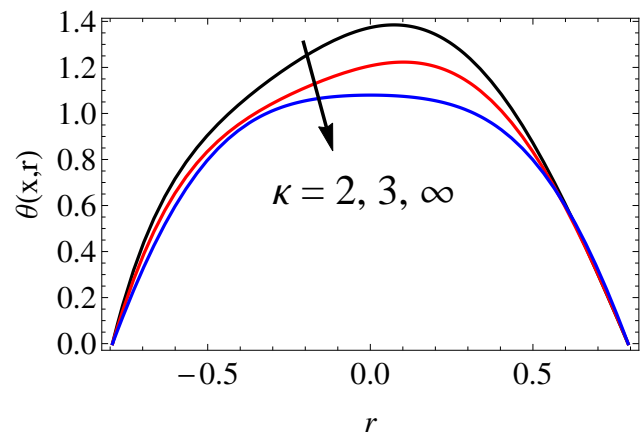

(a)

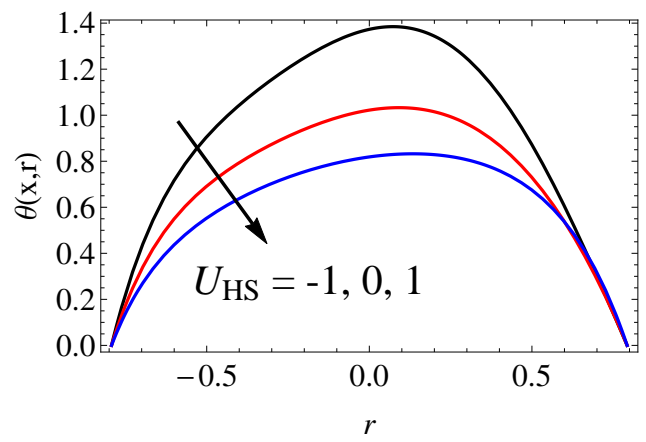

(c)

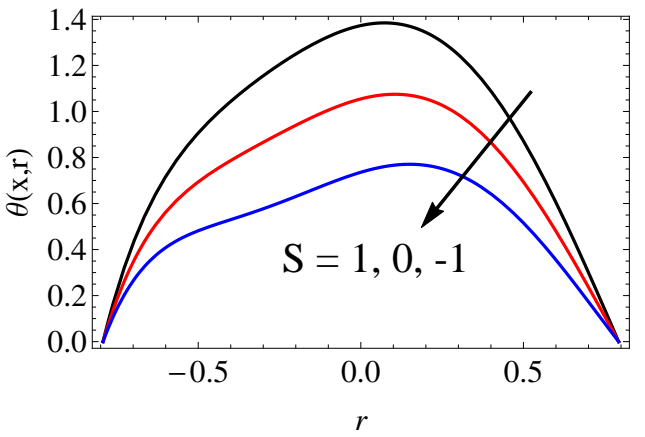

(b)

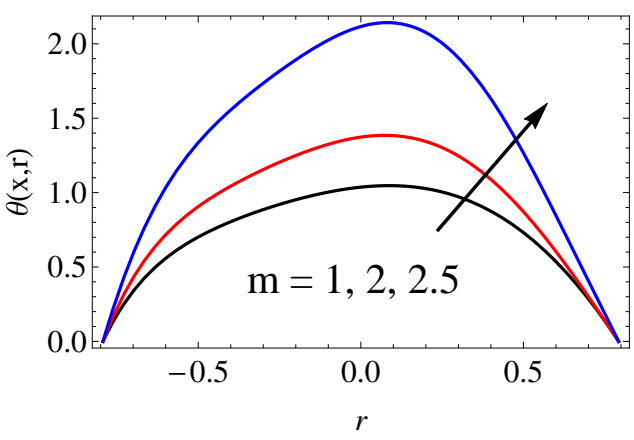

(d)

Figure 3: Temperature profiles for variation in different parameters (a) Brinkman Number $B r(b)$ joule heating parameter $S$ (c) electroosmotic velocity $U_{H S}$, (d) inverse EDL thickness parameters $m$, with other parameters $\phi=0.6, \kappa=2, Q_{T}=1.2, U_{H S}=-1, E c=1, \operatorname{Pr}=1, G r=1, G c=1$, $N t=0.5, N b=0.5, m=2, R_{\zeta}=1, x=0.3, t=1$. 
accelerate the flow in the lower micro-channel half space. The re-distribution of momentum in the regime also results in a deceleration for the same value of $U_{H S}$ in the upper micro-channel half space. Conversely for axial electrical field orientated in the reverse $x$-direction, negative $E_{x}$ results in a positive $U_{H S}$ value which effectively produces a negative electro-osmotic body force, this leads to strong deceleration in the lower micro-channel half space and a corresponding (but weaker) acceleration in the upper micro-channel half space. The case of vanishing electrical field $\left(E_{x} \rightarrow 0\right.$ associated with vanishing $\left.U_{H S}\right)$ naturally produces profiles which lie between the other two cases and corresponds to non-electrical curved micro-channel flow. It is also noteworthy that as $U_{H S}$ changes from negative to positive values (positive to negative electrical field, respectively) there is a displacement in peak axial velocity from the lower half space into the upper half space i.e. the profiles are skewed towards the upper curved wall. Inspection of Fig. 2(c) reveals that with a substantial increment in inverse EDL thickness parameter $(m)$ i.e. electro-osmotic parameter, there is a considerable boost in axial velocity in the lower micro-channel half space through the majority of this microchannel zone. Conversely the same elevation in $\mathrm{m}$ results in a deceleration in upper microchannel half space although this trend is not fully enforced for some distance beyond the centerline i.e. the acceleration in the upper half space overlaps partially into the lower micro-space and thereafter the retardation is observed with increasing $\mathrm{m}$ values. The electro-osmotic parameter is inversely proportional to the Debye length or characteristic thickness of the electrical double layer (EDL). A decrease in Debye length i.e. increasing $\mathrm{m}$ value, elevates electrical potential, as elaborated in Smith et al. [57]. The electrical potential is depleted systematically with elevation in Debye length and this is attributable to the enhanced quantity of ions which replace counter ions with further distance from the charged surfaces (micro-channel walls). Debye length emerges in electroosmosis as a crucial parameter which can be exploited to regulate the electrical potential distribution. By manipulation of the inverse EDL thickness parameter, $m$, the momentum and therefore velocity distribution may be controlled effectively. In Fig. 2(d) an elevation in positive value of the zeta potential ratio $R_{\zeta}\left(=\zeta_{1} / \zeta_{2}\right)$ there is a considerable acceleration in the flow in the lower microchannel half space whereas with an increase in negative zeta potential, significant flow deceleration is induced in the same zone. The opposite effect is induced in the upper microchannel half space i.e. negative zeta potential ratio generates acceleration whereas positive zeta potential ratio produces strong retardation. However 
negative values of axial velocity are never computed indicating that back flow (reversal of flow) is never induced anywhere in the micro-channel. $R_{\zeta}$ defines the ratio of the zeta potentials imposed at the upper and lower microchannel walls. Flow stability is ensured with higher zeta potential ratio. Since nanofluids are colloids with nano-particles suspended in the ionic base fluid, electrical stability is produced with high zeta potential (negative or positive) are therefore electrically-stabilized. With lower zeta potential ratio, coagulation or flocculation may arise which is undesirable. Effectively zeta potential ratio provides a useful quantification of the characterization of double-layer properties in micro-channel flows. It provides insight into the extent of electrostatic repulsion between adjacent, similarly charged particles in a nanofluid suspension or indeed in any other ionic colloid. For molecules and particles that are small enough, a high zeta potential will confer stability, i.e., the solution or dispersion will resist aggregation. When the potential is small, attractive forces may exceed this repulsion and the dispersion may break and flocculate, as noted by, among others, Murshed et al. [58].

Figs. 3(a)-3(d) illustrate the evolution in radial temperature distribution in the micro-channel with a change in $(a)$ curvature parameter $\kappa,(b)$ Joule heating (electrical dissipation) $S,(c)$ Helmholtz-Smoluchowski velocity $U_{H S}$, $(d)$ inverse EDL thickness parameter $m$. It is immediately evident that a much more homogenous distribution in temperature profiles is obtained as compared with axial velocity profiles i.e. inverse parabolic distributions are computed across the micro-channel cross-section, with peak temperatures always arising near the core zone. Fig. 3(a) shows that with increasing curvature effect (smaller values of the curvature parameter), there is a significant enhancement in temperatures across the width of the micro-channel. Straighter micro-channel geometries therefore produce significant cooling in the regime. Thermal convection heat transfer is aided with curvature of the micro-channel. The maximum temperature arises for a straight microchannel at the centreline, whereas with increasingly curved geometries this peak migrates into the upper half space. Fig. 3(b) indicates that negative Joule heating $(S=-1)$ generates a notable reduction in temperature across the microchannel whereas positive Joule heating $(S=1)$ manifests in a marked boost in temperatures. Ionic charges are localized at the walls and not in the bulk of the nanofluid. The presence of the negative ion charges on the wall surfaces mobilizes a concentration gradient of positively charged ions near the wall surfaces, but no tangible injection of charges along the central line of the channel. This results in an electrical potential distribution in the 


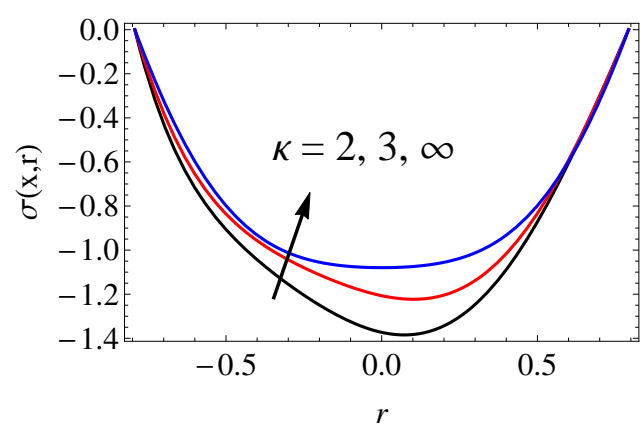

(a)

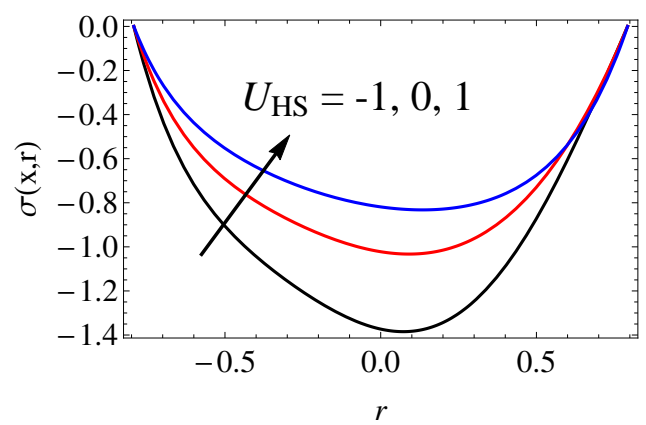

(c)

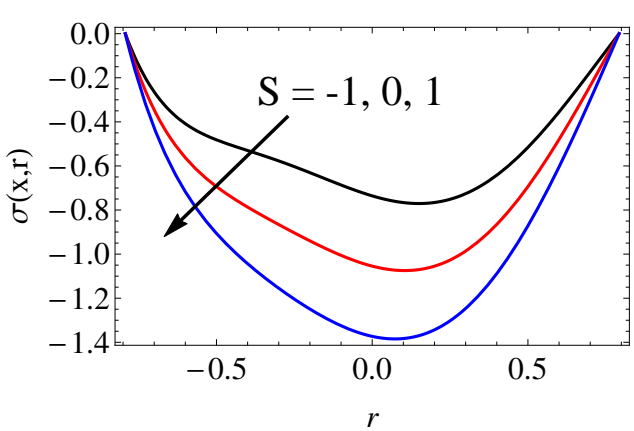

(b)

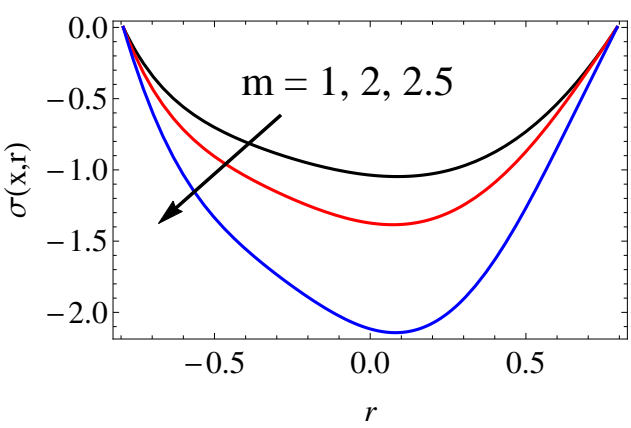

(d)

Figure 4: Concentration profiles for variation in different parameters (a) Brinkman Number $B r$ (b) joule heating parameter $S$ (c) electroosmotic velocity $U_{H S}$, (d) inverse EDL thickness parameters $m$, with other parameters $\phi=0.6, \kappa=2, Q_{T}=1.2, U_{H S}=-1, E c=1, \operatorname{Pr}=1, G r=1, G c=1$, $N t=0.5, N b=0.5, m=2, R_{\zeta}=1, x=0.02, t=0$. 
electrolyte which is known as the electric double layer (EDL). When electrical field (axial) is increased i.e. with greater Joule parameter (for constant temperature difference), the electro-osmotic body force is also enhanced. The change is inhibitive for negative $S$ values and assisting for positive $S$ values resulting respectively in cooling and heating of the micro-channel. The Joule heating term appears in the energy conservation equation (18), and although linear, exerts a considerable influence on the electro-osmotic heat transfer. The Joule heating parameter, $S=\left(\sigma_{e} E_{x}^{2} a^{2}\right) / K T_{0}$. Clearly it is quadratically linked to the electrical field, $E_{x}$ and is also directly proportional to electrical conductivity of the nanofluid, $\sigma_{e}$. With positive values of $S$ the nanofluid is energized similar to a heat source whereas with negative values of $S$ it is cooled. Electrical energy dissipated is converted to heat when $S>0$ and is removed from the nanofluid when $S<0$. The case where Joule heating is neglected corresponds to $S=0$. Neglect of Joule heating therefore clearly leads to unrealistic temperature predictions since it under predicts or overpredicts temperatures depending on the nature of the flow. Cruz et al. [59] have elaborated on the sensitivity of electro-osmotic heat transfer to electrical conductivity and also the relationship with nanoparticle stability. These are also important considerations in practical smart electro-nanofluid pumps. Glory et al. [60] have also emphasized the inter-dependence of heat transfer and electro-conductive properties in nanofluids. Generally stable solutions have been obtained for the scenario of equal thermal and species buoyancy forces, visualized in Fig. 3(b). Fig. 3(c) shows that an increase in maximum electroosmotic velocity $\left(U_{H S}\right)$ generates a reduction in temperature whereas a decrease induces a significant heating in the ionic nanofluid. Positive $U_{H S}$ (reverse axial electrical field, $E_{x}$ ) inhibits the momentum diffusion and also thermal diffusion whereas negative $U_{H S}$ (aligned axial electrical field, $E_{x}$ ) encourages thermal diffusion leading to an elevation in temperatures. No switch in profiles is computed across the micro-channel span. Fig. 3(d) indicates that increasing positive values of inverse EDL thickness parameter $(m)$ i.e. electro-osmotic parameter, leads to a consistent enhancement in temperatures of the ionic nanofluid across the span. Heating is therefore encouraged with lower electrical double layer thickness whereas cooling is induced with higher electrical double layer thickness (lower inverse EDL thickness parameter value) in the regime.

Figs. 4(a)-4(d) depict the radial nano-particle concentration profiles, $\sigma(x, r)$, for different values of $(a)$ curvature parameter $\kappa$, (b) Joule heating (electrical dissipation) $S,(c)$ Helmholtz-Smoluchowski velocity $U_{H S},(d)$ in- 
verse EDL thickness parameter $m$. Significantly different profiles (parabolas) are computed as compared with the temperature profiles (inverse parabolas) in Fig 3. In all cases maximum nano-particle concentrations are computed at the micro-channel walls and the minimum values are observed in the vicinity of the micro-channel centerline. An increase in curvature parameter, $\kappa$, (fig. $4(\mathrm{a})$ ) is observed to considerably reduce nano-particle concentration magnitudes in the ionic nanofluid, which is the reverse effect to that induced in the temperature field. With greater curvature of the micro-channel (lower $\kappa$ ) species (nano-particle) diffusion is inhibited and nano-particle concentration magnitudes are depleted across the micro-channel span. The maximum nanoparticle concentration is obtained for the case of a straight micro-channel $\kappa \rightarrow \infty)$. Geometry there has a profoundly different influence on species diffusion as compared with heat diffusion. Fig. 4(b) shows that with negative Joule heating parameter $(S=-2)$ the nano-particle concentrations are boosted whereas they are suppressed with positive Joule heating parameter $(S=2)$. Again, this is the reverse behavior to that computed for the temperature distributions (Fig. 3(b)). As noted earlier, with positive values of $S$ the nanofluid is energized similar to a heat source whereas with negative values of $S$ it is cooled. Thermal diffusion is encouraged with positive $S$ whereas nano-particle diffusion is inhibited. The addition of thermal energy with positive Joule electrical energy dissipation is counter-productive for the transport of nano-particles. The implication is that negative Joule heating is advantageous for stimulating enhanced species diffusion in real systems. It has been shown by Zayid [61] that the electrical conductivity of nanofluids shows enhancement with an increase in the concentration of nanoparticles. It is therefore an important consideration in future work to consider variable electrical conductivity of the nanofluid as this may provide an improved insight into the collective influence of electrical field and the type and doping of nanofluids with different nano-particles on heat and mass transfer characteristics. It is also important to note that the Joule heating parameter $\left(S=\left(\sigma_{e} E_{x}^{2} a^{2}\right) / K T_{0}\right)$ is also inversely proportional to thermal conductivity of nanofluid, although in the current study this is not examined. In Fig. 4(c), increasing maximum electro-osmotic velocity (positive $U_{H S}$ corresponding to reverse axial electrical field, $E_{x}$ ) induces a notable reduction in nano-particle concentration whereas decreasing maximum electro-osmotic velocity (negative $U_{H S}$ i.e. positively aligned axial electrical field) leads to significant elevation in nano-particle concentrations. Again, therefore the orientation of axial electrical field, as simulated via $U_{H S}$ has a major influence on the distribution 
of nano-particles in the regime. Fig. 4d demonstrates that there is a substantial suppression in nano-particle concentrations in the ionic nanofluid (across the micro-channel span) with larger values of electro-osmotic parameter $(\mathrm{m})$ i.e. a decrease in electrical double layer thickness. As described earlier, $m$ is inversely proportional to the Debye length or characteristic thickness of the electrical double layer (EDL). Lower values of Debye length i.e. increasing $m$ value, elevate electrical potential, which encourages the replacement of ions with counter ions in the regime. This counter-acts the diffusion of nano-particles, as also noted by Zayid [61] and leads to smaller values of nano-particle concentration. The effect also noted by Smith et al. [57] that is maximized with further distance from the charged surfaces (micro-channel walls) i.e. in the core zone of the micro-channel, leading to a minimization in nano-particle concentrations around the micro-channel centerline.

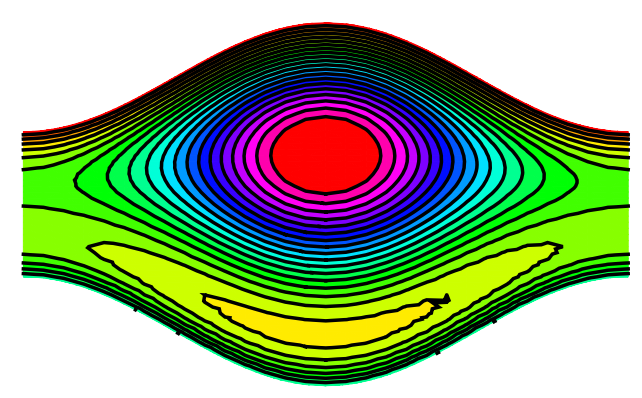

(a) $\kappa=2$

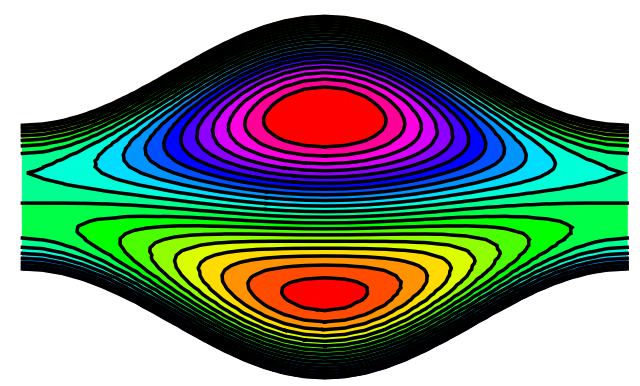

(c) $\kappa=10$

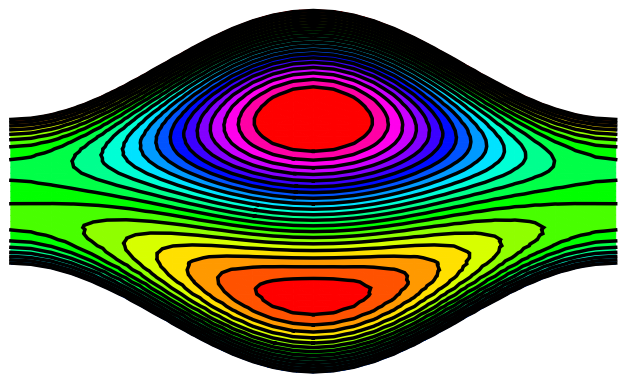

(b) $\kappa=5$

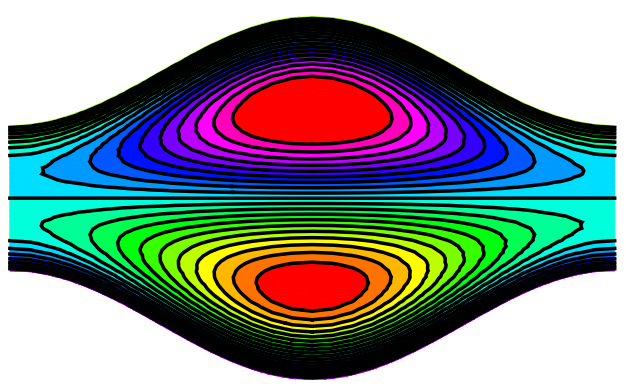

(d) $\kappa->\infty$

Figure 5: Stream lines for channel curvature in wave frame of reference. The other parameters are $\phi=0.6, \kappa=2, Q_{T}=1.2, U_{H S}=-1, E c=1, \operatorname{Pr}=1$, $G r=1, G c=1, N t=1, N b=1, m=2, R_{\zeta}=1, x=0.3, S=1$. 


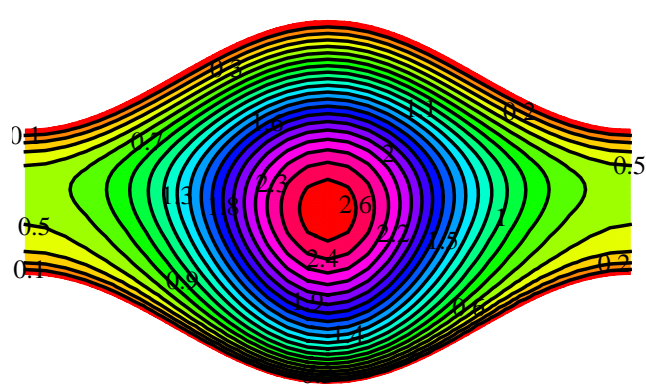

(a) $\kappa=1.5$

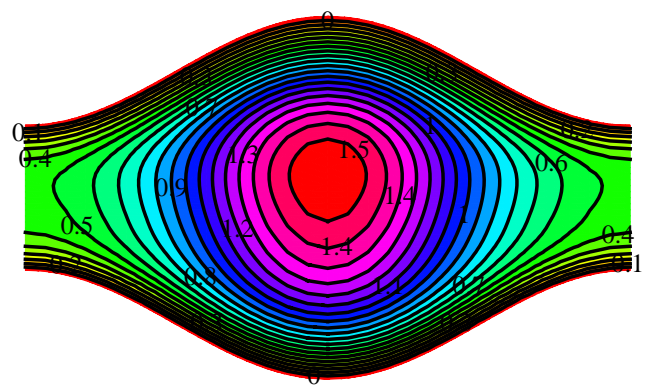

(c) $\kappa=5$

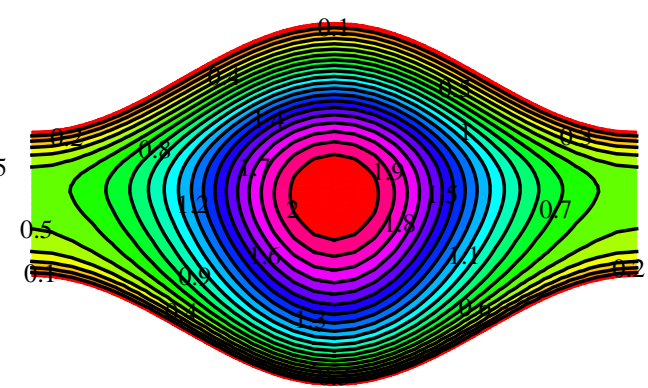

(b) $\kappa=2$

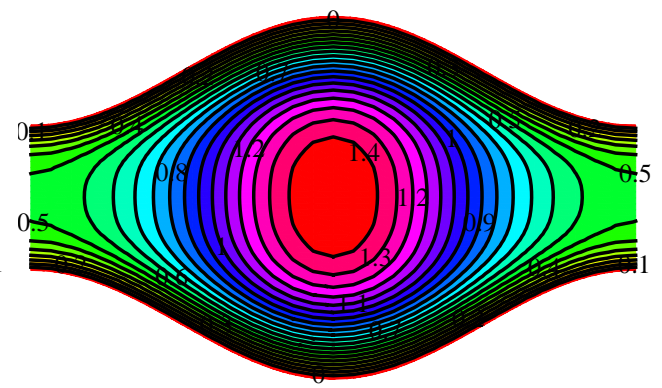

(d) $\kappa=10$

Figure 6: Temperature contours for channel curvature. The other parameters are $\phi=0.6, \kappa=2, Q_{T}=1.2, U_{H S}=-1, E c=1, \operatorname{Pr}=1, G r=1, G c=1$, $N t=1, N b=1, m=2, R_{\zeta}=1, x=0.02, S=1$. 


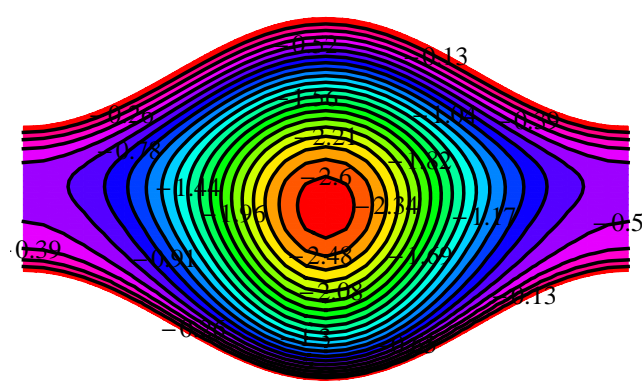

(a) $\kappa=1.5$

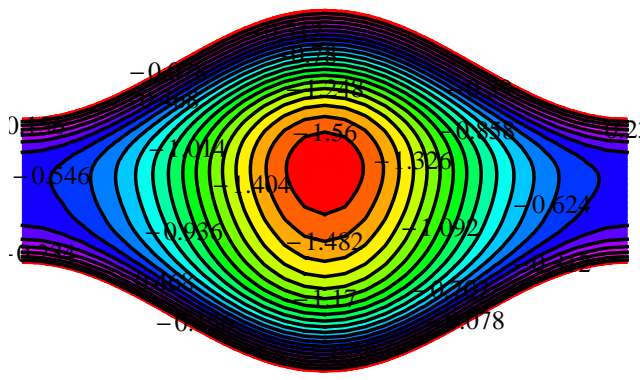

(c) $\kappa=5$

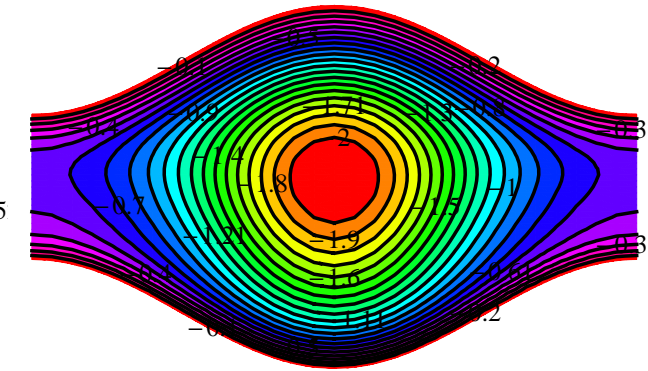

(b) $\kappa=2$

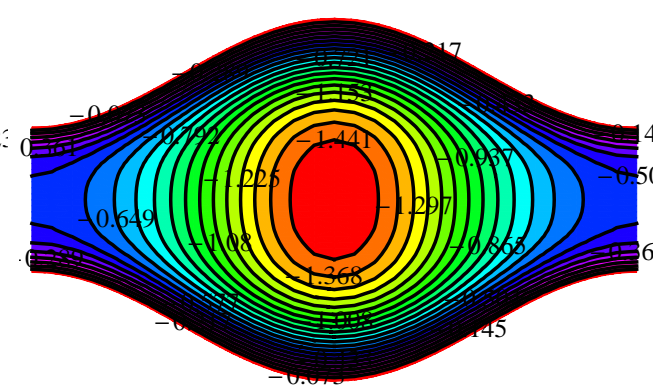

(d) $\kappa=10$

Figure 7: Nanoparticles concentration contours for channel curvature. The other parameters are $\phi=0.6, \kappa=2, Q_{T}=1.2, U_{H S}=-1, E c=1, \operatorname{Pr}=1$, $G r=1, G c=1, N t=1, N b=1, m=2, R_{\zeta}=1, x=0.02, S=1$. 
Figs. 5-7 illustrate respectively the streamline, temperature and nanoparticle concentration contours in the peristaltic microchannel flow with variation in the conduit curvature parameter $(\kappa)$. Figs. $5(\mathrm{a})-5(\mathrm{~d})$ show that higher curvature parameter (associated with an increasingly straighter geometry) modifies the dominant single bolus structure in the upper microchannel half space into a dual structure. The smaller lower bolus in Fig. 5(a) (strong curvature, $\kappa=2$ ) is expanded considerably with increasing $\kappa$ values (Fig $5(\mathrm{~b}), \kappa=5$ and Fig. 5(c), $\kappa=10)$ and progressively more elliptic boluses are synthesized in the two half spaces of the micro-channel. Vorticity structure is therefore strongly influenced by a decrease in curvature of the microchannel. A more symmetric streamline distribution is achieved for straight channels (Fig. 5(d)), although there is a still a marginally larger bolus in the upper half space. Figs. 6(a)-6(d) illustrate that only a single temperature (isotherm) structure is computed in the entire micro-channel, as opposed to the dual vortex structure for the streamlines (Figs. 5(a)-5(d)). As curvature parameter is enhanced this central zone shrinks laterally (Fig. 6(a)) and extends vertically (Figs 6(b), 6(c)) eventually leading to a more circular structure (Fig. 6(d)). The isotherms are compressed laterally and expanded vertically therefore for less curved micro-channels. Dual symmetry is however generally sustained for all values of curvature parameter except for the intermediate case of $\kappa=5$ where the upper zone circular isotherms is more dilated than the lower constricted zone. Heat transfer characteristics are therefore confirmed to be modified significantly in the micro-channel with curvature. Inspection of Figs 7(a)-7(d) reveals that the iso-solute (nano-particle concentration contours), exhibit a similar topology to the temperature contours. A solitary bolus is observed at all values of curvature parameter, $(\kappa)$. However with increasing values, there is a more dramatic modification in bolus structure. The generally circular structure at the centre of the micro-channel progressively morphs to a vertically biased oval structure with an increase in $(\kappa)$ from 1.5 (strongly curved micro-channel) through 2, 5 to 10 (an almost straighter microchannel). Evidently there is an intensification in the proximity of the concentration contours, at both the upper and lower microchannel walls accompanying the transition to an oval bolus structure with a relaxation in concentration contours in the lateral direction. Overall the impact of curvature on all flow characteristics in the peristaltic regime is considerable. Figs. 8(a) and 8(b) illustrate axial distribution of average entropy generation rate, $E_{S, A v g}$. with variation in curvature parameter $(\kappa)$ and Helmholtz-Smoluchowski velocity $\left(U_{H S}\right)$. An oscillatory behaviour is com- 


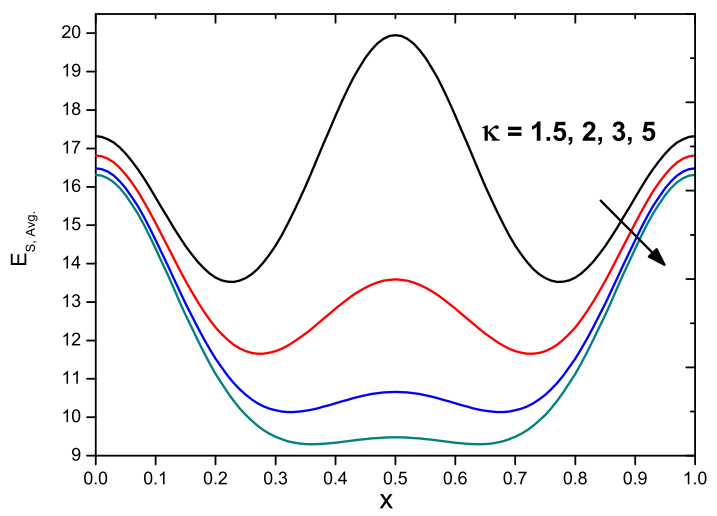

(a)

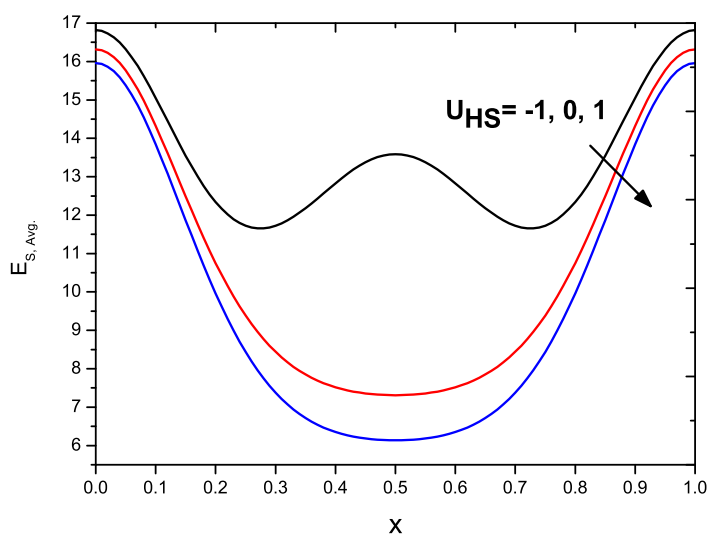

(b)

Figure 8: Average entropy generation rate $E_{S, A v g}$. vs axial length. The other parameters are $\phi=0.6, \kappa=2, Q_{T}=1.2, U_{H S}=-1, E c=1, \operatorname{Pr}=1$, $G r=1, G c=1, N t=1, N b=1, m=2, R_{\zeta}=1, S=0.5, \Gamma=1, \Omega=1$, $\Lambda=1, \Upsilon=0.5$. 
puted from the inlet to the outlet of the finite micro-channel. High values of average entropy generation rate arise at both extremities. In fig. 8(a), the peak value of average entropy generation rate, $E_{S, A v g}$. is observed in the mid-region of the micro-channel. With increasing curvature parameter $(\kappa)$ there is a significant depression in $E_{S, A v g}$. More curved geometries (lower value) therefore achieve greater average entropy generation rates compared with straighter micro-channel geometries. Fig. 8b shows that average entropy generation rate is enhanced with negative Helmholtz-Smoluchowski velocity $\left(U_{H S}\right)$ whereas it is depleted for positive Helmholtz-Smoluchowski velocity. Entropy generation is therefore elevated with aligned axial electrical field $\left(E_{x}\right)$ whereas it is suppressed in the micro-channel with reverse axial electrical field.

Figs. 9(a) and 9(b) depict the evolution in average entropy generation rate, $E_{S, A v g}$. with time-averaged flow rate $\left(Q_{T}\right)$ for different values of $(a)$ inverse EDL thickness parameter $m$, $(b)$ zeta potential ratio $R_{\zeta}$. There is a monotonic increase in entropy generation rate with volumetric flow rate. Fig 9(a) shows that entropy generation rate is strongly elevated (for equal potential at the upper and lower micro-channel walls i.e. $R_{\zeta}=1$ with inverse EDL thickness parameter $(m)$ indicating that smaller Debye lengths and higher electrical potential encourage the production of entropy. The opposite effect i.e. entropy generation reduction, which is desirable from a thermodynamic optimization viewpoint, is achieved by decreasing inverse EDL thickness parameter $m$ i.e. increasing Debye length and lower electrical potential. Fig. 9(b) indicates that for relatively large inverse EDL thickness parameter $(m=2)$ entropy generation rate, $E_{S, A v g .}$, is increased with positive increase in zeta potential ratio; however a markedly greater enhancement is generated with greater negative values of zeta potential ratio. This indicates that the polarity of electrical potential at the micro-channel walls has a considerable impact on entropy generation in the system. Larger negative difference in zeta potential ratio leads to an undesirable increase in entropy generation. Entropy generation is therefore minimized with smaller zeta potential ratio when the polarity of the micro-channel walls is the same. Figs. 10(a)-10(d) present the radial Bejan number $(B e)$ distributions with a change in $(a)$ curvature parameter $\kappa,(b)$ Helmholtz-Smoluchowski velocity $U_{H S},(c)$ inverse EDL thickness parameter $m,(d)$ zeta potential ratio $R_{\zeta}$. With decreasing curvature parameter (stronger curved micro-channel), there is initially a reduction in Bejan number in the vicinity of the lower microchannel wall; however this is superseded quickly with a strong elevation in 


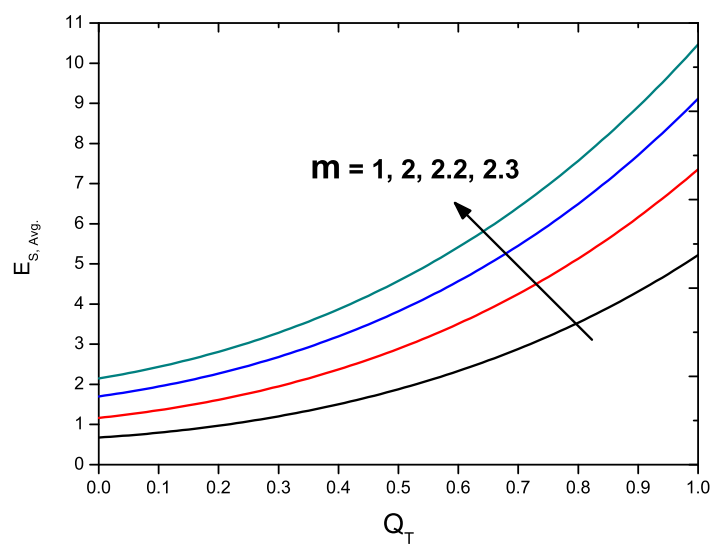

(a)

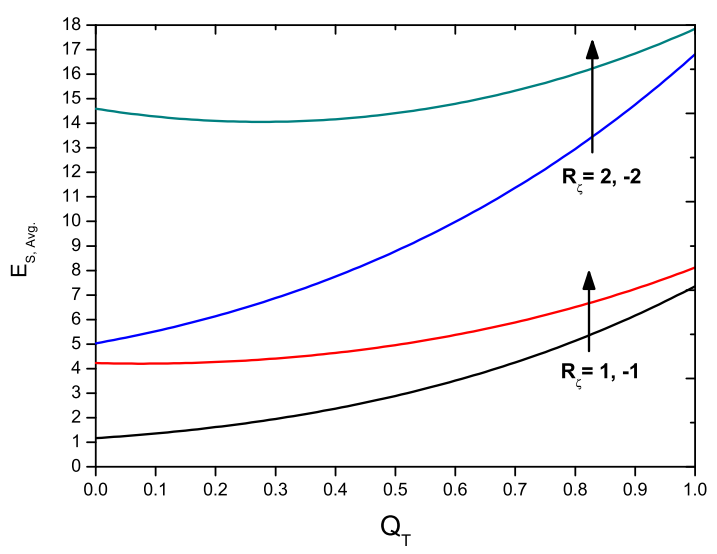

(b)

Figure 9: Average entropy generation rate $E_{S, A v g}$. with the time averaged flow rate. The other parameters are $\phi=0.6, \kappa=2, U_{H S}=-1, E c=1$, $\operatorname{Pr}=1, G r=1, G c=1, N t=1, N b=1, m=2, R_{\zeta}=1, x=0.3, S=0.5$, $\Gamma=1, \Omega=1, \Lambda=1, \Upsilon=0.5$. 


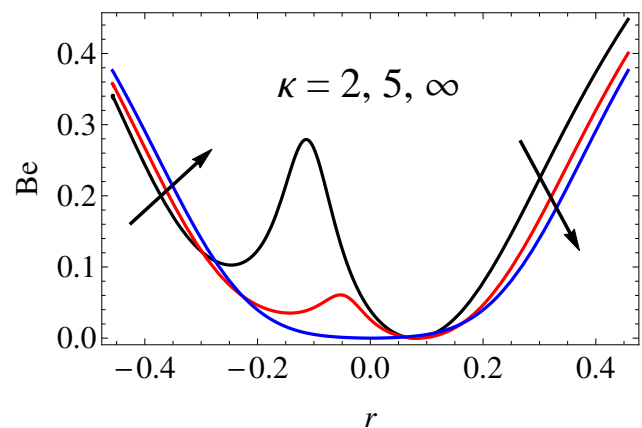

(a)

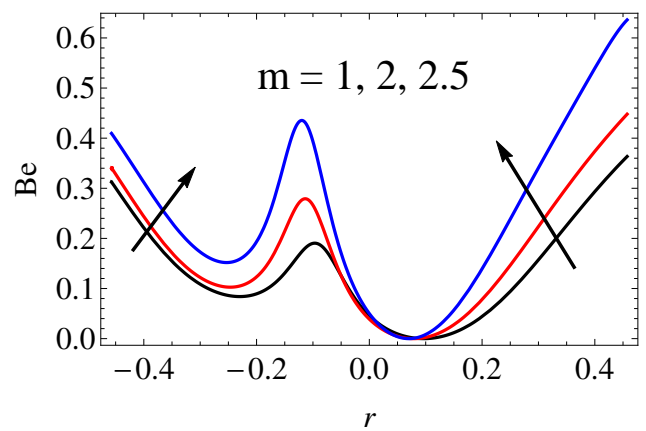

(c)

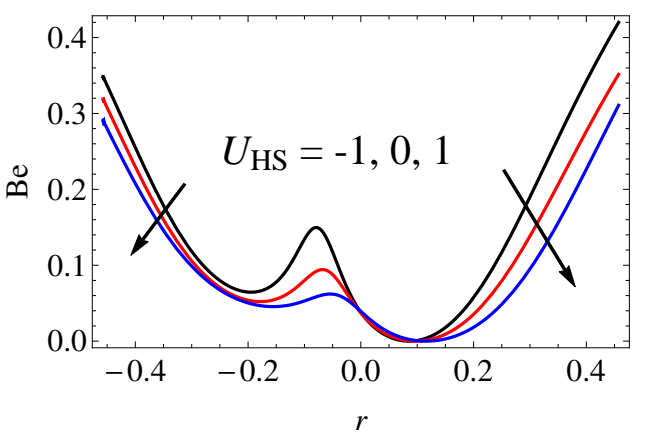

(b)

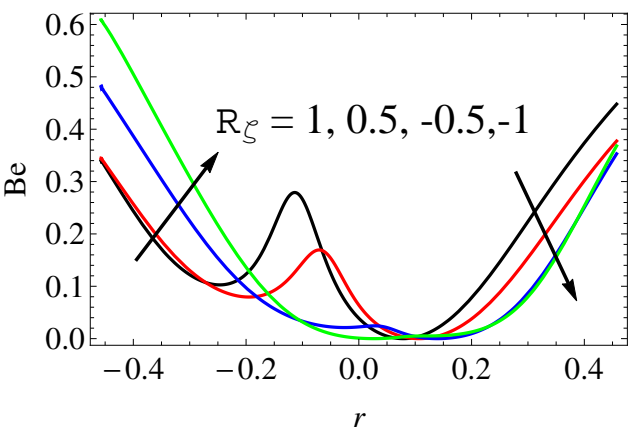

(d)

Figure 10: Bejan number with the radial distance. The other parameters are $\phi=0.6, \kappa=2, U_{H S}=-1, E c=1, \operatorname{Pr}=1, G r=1, G c=1, N t=1$, $N b=1, m=2, R_{\zeta}=2, x=0.1, S=0.5, \Gamma=1, \Omega=1, \Lambda=1, \Upsilon=0.5$. 
Bejan number across the remainder of the micro-channel span (radial coordinate) with decreasing curvature. The peak Bejan number arises in the lower micro-channel half space, near the centre-line and this zone also corresponds to the widest disparity in Bejan numbers with curvature effect. In the upper micro-channel half space although lower curvature parameter still attains the highest Bejan number, the influence is less prominent. Fig. 10b shows that with negative Helmholtz-Smoluchowski velocity $\left(U_{H S}\right)$ i.e. positive aligned axial electrical field, larger values of Bejan number are computed across the micro-channel span whereas with positive Helmholtz-Smoluchowski velocity $\left(U_{H S}\right)$ i.e. reverse axial electrical field, much smaller values of Bejan number are observed. Fig. 10(c) indicates that Bejan number is is generally increased with greater values of inverse EDL thickness parameter (smaller Debye lengths) in the radial direction. Although initially the influence of negative zeta potential ratio, $R_{\zeta}$, (Fig. $10(\mathrm{~d})$ ) is to significantly increase Bejan number (closer to the lower wall of the micro-channel); however as we progress across the micro-channel, there is a strong decrease in Bejan number. In the central zone and the upper micro-channel half space conversely a positive zeta potential ratio, $R_{\zeta}$, boosts the Bejan number. To understand the entropy generation mechanisms, it is necessary to consider the contribution of heat transfer to the overall irreversibility. For this purpose, the Bejan number is used which is an alternative irreversibility parameter. As noted earlier, Bejan number defines the ratio of irreversibility due to heat transfer to the total irreversibility. When $B e=1$, the irreversibility due to heat transfer dominates and when $B e=0$ the irreversibility due to fluid friction and electrical field dominates. It is clear, when $B e=0.5$, the contribution to entropy due to heat transfer is equal to the sum of electrical field and fluid friction effects. Clearly optimum Bejan numbers can be achieved via careful manipulation of the electrical potential at the walls of the micro-channel and this is beneficial in electro-osmotic nanofluid pump design.

\section{Conclusions}

A mathematical study of entropy generation in peristaltic propulsion of an electro-osmotic ionic nanofluid in a curved deformable microchannel under the action of an axial electrical field has been presented. Thermal and species (nano-particle) buoyancy effects and Soret and Dufour cross-diffusion effects have also been computed. A dilute nanofluid has been considered with Brownian motion and thermophoretic body forces present. Joule elec- 
trical dissipation has also been included in the model in addition to thermal and species (nano-particle) buoyancy effects. The reduced non-dimensional conservation equations have been solved analytically after employing lubrication approximations and low zeta potential (Debye-Hückel linearization). Numerical evaluation of these closed-form solutions have been extracted with the symbolic software, Mathematica 9 via the NDSolve algorithm. Velocity, temperature, nano-particle concentration, streamline, isotherm, iso-solutal (nano-particle concentration contour), average entropy generation rate and Bejan number distributions have been plotted. The influence of curvature parameter, maximum electroosmotic velocity (Helmholtz-Smoluchowski velocity), inverse EDL thickness parameter, zeta potential ratio and Joule heating parameter on transport characteristics have been studied. The computations have shown that:

1. Entropy generation rate is increased with positive increase in zeta potential ratio; however a more substantial enhancement is computed with greater negative values of zeta potential ratio.

2. Bejan number is reduced for more curved micro-channels (lower values of curvature parameter) whereas it is generally elevated with greater values of inverse EDL thickness parameter (lower Debye lengths) in the radial direction.

3. Average entropy generation rate is strongly boosted along the axis of the curved channel with increasing curvature and for negative HelmholtzSmoluchowski velocity whereas it is depleted for positive HelmholtzSmoluchowski velocity.

4. With lower values of curvature parameter (more curved micro-channel geometry) the average entropy generation rate peaks at a central location along the micro-channel whereas for lower curvatures the peaks are located at the extremities of the microchannel i.e. entry and exit.

5. There is a monotonic increase in entropy generation rate with volumetric flow rate.

6. Temperatures are elevated with positive Joule heating and reduced with negative Joule heating whereas the converse response is computed for the nano-particle concentration distributions. 
7. With greater curvature parameter (corresponding to a progressively straighter microchannel), the streamline patterns reveal that the dominant single bolus structure in the upper microchannel half space morphs into a dual structure.

8. With greater curvature parameter (corresponding to a progressively straighter microchannel), the isotherms and iso-solute patterns indicate that the closed contours around a stagnation point is elongated in the vertical direction and transitions from a circular structure to an oval one.

9. Increasing curvature parameterleads to a retardation in axial velocity in the lower micro-channel half space whereas it generates strong acceleration in the upper micro-channel half space.

10. Increasing positive Joule heating parameter elevates temperatures whereas it suppresses nano-particle concentration magnitudes across the microchannel span; increasing negative Joule heating parameter results in the converse behaviour.

11. Increasing positive Helmholtz-Smoulochowski velocity (corresponding to reverse axial electrical field) reduces temperatures whereas it accentuates nano-particle concentration values, with these trends sustained across the microchannel.

The current simulations have however provided a good insight into the phenomena intrinsic to electro-osmotic nanofluid pumping with a biologicallyinspired (peristaltic) mechanism.

\section{Appendix A.}

The vector operators in the governing equations are

$$
\begin{gathered}
(\bar{V} \cdot \nabla)=\frac{R u}{(r+R)} \frac{\partial}{\partial x}+v \frac{\partial}{\partial r} \\
\nabla^{2}=\left(\frac{R}{r+R}\right)^{2} \frac{\partial^{2}}{\partial x^{2}}+\frac{1}{r+R} \frac{\partial}{\partial r}+\frac{\partial^{2}}{\partial r^{2}},
\end{gathered}
$$




\section{Appendix B.}

The transformations between the wave and the laboratory frames, in dimensionless form, are:

$$
X=x-t, Y=r, U=u-1, V=v, q=F-2 h, \Psi=\psi-r
$$

where the left hand side parameters are in the wave frame and the right hand side parameters are in the laboratory frame.

The dimensionless volume flow rate in laboratory frame is defined as

$$
F(x, t)=\int_{-h}^{h} u(x, r, t) d r
$$

and the time averaged flow rate over one period, yielding

$$
Q_{T}(x)=\int_{0}^{1} F(x, t) d t .
$$

The relation between volume flow rate and time averaged flow rate is

$$
F(x, t)=Q_{T}+2(h(x, t)-1) .
$$

\section{References}

[1] Z. Zhang, P. Li, X.-Y. Kong, G. Xie, Y. Qian, Z. Wang, Y. Tian, L. Wen, L. Jiang, Bioinspired heterogeneous ion pump membranes: unidirectional selective pumping and controllable gating properties stemming from asymmetric ionic group distribution, J. Am. Chem. Soc 140(3) (2018) 1083-1090.

[2] J. R. Burns, A. Seifert, N. Fertig, S. Howorka, A biomimetic dna based channel for the ligand controlled transport of charged molecular cargo across a biological membrane, Nat. Nanotechnol. 11 (2016) 152-156.

[3] O. A. Beg, M. F. MdBasir, M. J. Uddin, A. I. MdIsmail, Numerical study of slip effects on asymmetric bioconvective nanofluid flow in a porous microchannel with an expanding/contracting upper wall using buongiorno's model, J Mech Med Biol. 17(5) (2017) 1750059. 
[4] J. Zhang, Biomimetic multifunctional nanochannels based on the asymmetric wettability of heterogeneous nanowire membranes, Adv. Mater. 26 (2014) 1071-1075.

[5] P. Jiang, G. Huang, Y. Zhu, R. Xu, Z. Liao, T. Lu, Experimental investigation of biomimetic self-pumping and self-adaptive transpiration cooling, Bioinspir Biomim. 12(5) (2017) 056002.

[6] H. Zhang, G. A. Aggidis, Nature rules hidden in the biomimetic wave energy converters, Renew Sust Energ Rev 97 (2018) 28-37.

[7] D. M.Tiede, K. L. Mardis, X. Zuo, X-ray scattering combined with coordinate-based analyses for applications in natural and artificial photosynthesis, Photosynth Res. 102(2-3) (2009) 267-279.

[8] P. Bandyopadhyay, J. Castano, J. Rice, R. Philips, W. Nedderman, W. Macy, Low speed maneuvering hydrodynamics of fish and small underwater vehicles, ASME J Fluids Eng. 119 (1997) 136-144.

[9] S. Heo, T. Wiguna, H. C. Park, N. S. Goo, Effect of an artificial caudal fin on the performance of a biomimetic fish robot propelled by piezoelectric actuators, J. Bionic Engineering 4 (2007) 151-158.

[10] B. Bhushan, Y. C. Jung, Wetting, adhesion and friction of superhydrophobic and hydrophilic leaves and fabricated micro/nanopatterned surfaces, J. Phys. Condens Matter 20 (2008) 225010.

[11] M. Y. Jaffrin, A. H. Shapiro, Peristaltic pumping, Ann. Rev. Fluid Mech. 3 (1971) 13-37.

[12] S. Seok, C. D. Onal, R. Wood, D. Rus, , S. Kim, Peristaltic locomotion with antagonistic actuators in soft robotics, in: IEEE International Conference on Robotics and Automation (ICRA), Anchorage, Alaska, USA, 2010, pp. 1228-1233.

[13] D. Kim, W. S. L. S. Shin, H. S. Rho, J. Dai, J. Y. Y. J. W. Hong, Quantitative analysis of pneumatically driven biomimetic micro peristalsis, Sci. Adv. Mater. 6(11) (2014) 2428-2434.

[14] M. Zhu, W. Xu, L. K. Cheng, Esophageal peristaltic control of a softbodied swallowing robot by the central pattern generator, IEEE/ASME Trans. Mechatron. 22 (2016) 91-98. 
[15] S. Dirven, J. Allen, W. X. L. K. Cheng, Soft-robotic esophageal swallowing as a clinically-inspired bolus rheometry technique, Meas. Sci. Technol. 28 (2017) 035701.

[16] M. R. J. Mohan, Boundary layer flow acceleration by paraelectric and peristaltic EHD effects of aerodynamic plasma actuators, Master's thesis, University of Tennessee, Knoxville, USA, 2004. Master's Thesis.

[17] M. A. Abbas, Y. Q. Bai, M. M. Rashidi, M. M. Bhatti, Application of drug delivery in magnetohydrodynamics peristaltic blood flow of nanofluid in a non-uniform channel, Chin. J. Phys. 16(04) (2016) 1650052 .

[18] M. M. Bhatti, A. Zeeshan, R. Ellahi, Heat transfer analysis on peristaltically induced motion of particle-fluid suspension with variable viscosity: clot blood model, Comput. Methods Programs. Biomed. 137 (2016) 115-124.

[19] M. M. Bhatti, A. Zeeshan, R. Ellahi, Endoscope analysis on peristaltic blood flow of sisko fluid with titanium magneto-nanoparticles, Comput. Biol. Med. 78 (2016) 29-41.

[20] M. M. Bhatti, A. Zeeshan, R. Ellahi, Simultaneous effects of coagulation and variable magnetic field on peristaltically induced motion of jeffrey nanofluid containing gyrotactic microorganism, Microvasc. Res. 110 (2017) 32-42.

[21] M. M. Bhatti, A. Zeeshan, R. Ellahi, O. A. Bég, A.Kadir, Effects of coagulation on the two-phase peristaltic pumping of magnetized prandtl biofluid through an endoscopic annular geometry containing a porous medium, Chin. J. Phys. 58 (2019) 222-234.

[22] S. I. Abdelsalam, M. M. Bhatti, New insight into aunp applications in tumour treatment and cosmetics through wavy annuli at the nanoscale, Sci. Rep. 9 (2019) 9:260(1-14).

[23] N. Manzoor, K. Maqbool, O. A. Bég, S. Shaheen, Adomian decomposition solution for propulsion of dissipative magnetic jeffrey biofluid in a ciliated channel containing a porous medium with forced convection heat transfer, Heat Transfer Asian Res. 48(2) (2018) 556-581. 
[24] X. Guo, J. Zhou, H. Xie, Z. Jiang, Mhd peristaltic flow of fractional jeffrey model through porous medium, Math Probl Eng. 2018 (2018) 6014082 .

[25] Y. AbdElmaboud, K. S. Mekheimer, S. I. Abdelsalam, A study of nonlinear variable viscosity in finite-length tube with peristalsis, Appl Bionics Biomech. 11 (2014) 197-206.

[26] J. C. Gómez-Blanco, F. J. Martnez-Reina, D. Cruz, J. B. Pagador, F. M. Sánchez-Margallo, F. Soria, Fluid structural analysis of urine flow in a stented ureter, Comput Math Methods Med. 2016 (2016) 5710798.

[27] S. U. S. Choi, J. A. Eastman, Peristaltic locomotion with antagonistic actuators in soft robotics, in: D. A. Singer, H. P. Wang (Eds.), ASME International Mechanical Engineering Congress \& Exposition, ASME Fluids Division, USA, 1995, pp. 99-105.

[28] M. A. M. Hassan, H. M. Abdel-Hameed, O. E. Mahmoud, Experimental investigation of the effect of nanofluid on thermal energy storage system using clathrate, ASME J Energy Resour Technol. 141(4) (2018) 042003(1-8).

[29] S. Kuharat, O. A. Bég, A. Kadir, M. Babaie, Computational fluid dynamic simulation of a solar enclosure with radiative flux and different metallic nano-particles, in: International Conference on Innovative Applied Energy (IAPE'19), The King's Centre, University of Oxford, United Kingdom, 2019.

[30] M. R. Islam, B. Shabani, G. Rosengarten, Nanofluids to improve the performance of pem fuel cell cooling systems: A theoretical approach, Appl Energ. 178 (2016) 660-671.

[31] R. Ellahi, The effects of mhd and temperature dependent viscosity on the flow of non-newtonian nanofluid in a pipe: analytical solutions, Appl. Math. Model. 37(3) (2013) 1451-1457.

[32] M. K. Nayak, N. S. Akbar, D. Tripathi, V. S. Pandey, Three dimensional mhd flow of nanofluid over an exponential porous stretching sheet with convective boundary conditions, Thermal Sci. Eng. Process 3 (2017) 133-140. 
[33] S. Rashidi, S. Akar, M. Bovand, R. Ellahi, Volume of fluid model to simulate the nanofluid flow and entropy generation in a single slope solar still, Renew. Energy 115 (2018) 400-410.

[34] J. Buongiorno, Convective transport in nanofluids, ASME J. Heat Transfer 128(3) (2005) 240-250.

[35] V. V. Polevoi, Electroosmotic phenomena in plant tissues, Biol. Bull. 30(2) (2003) 133-139.

[36] S. Bhansali, A. Vasudev, MEMS for Biomedical Applications, Woodhead Publishing Series in Biomaterials, USA, 2012.

[37] S. Chakraborty, Augmentation of peristaltic microflows through electroosmotic mechanisms, J. Phys. D 39 (2006) 5356-5363.

[38] D. Tripathi, A. Sharma, O. A. Bég, Electrothermal transport of nanofluids via peristaltic pumping in a finite micro-channel: effects of joule heating and helmholtz-smoluchowski velocity, Int. J. Heat Mass Transfer 111 (2017) 138-149.

[39] D. Tripathi, A. Sharma, O. A. Bég, Joule heating and buoyancy effects in electro-osmotic peristaltic transport of aqueous nanofluids through a microchannel with complex wave propagation, Adv Powder Technol 29 (2018) 639-653.

[40] S. Noreen, Quratulain, D. Tripathi, Heat transfer analysis on electroosmotic flow via peristaltic pumping in non-darcy porous medium, Thermal Sci. Eng. Process 11 (2019) 254-262.

[41] V. K. Narla, D. Tripathi, G. P. R. Sekhar, Time-dependent analysis of electroosmotic fluid flow in a microchannel, J Eng Math 114 (2019) $177-196$.

[42] V. K. Narla, D. Tripathi, O. A. Bég, Electro-osmosis modulated viscoelastic embryo transport in uterine hydrodynamics: mathematical modeling, J Biomech Eng-T ASME 141 (2019) 021003(1-10).

[43] A. Bejan, Entropy Generation through Heat and Fluid Flow, 1982. 
[44] N. S. Akbar, M. Shoaib, D. Tripathi, S. Bhushan, O. A. Bég, Analytical approach for entropy generation and heat transfer analysis of cntnanofluids through ciliated porous medium, J. Hydrodynamics 30(2) (2018) 1-11.

[45] J.Escandón, O.Bautista, F.Méndez, Entropy generation in purely electroosmotic flows of non-newtonian fluids in a microchannel, Energy 55 (2013) 486-496.

[46] L. Zhao, L. H. Liu, Entropy generation analysis of electro-osmotic flow in open-end and closed-end micro-channels, Int. J. Thermal Sciences 49 (2010) 418-427.

[47] H. Sato, T. Kawai, T. Fujita, M. Okabe, Two dimensional peristaltic flow in curved channels, Trans. Jpn. Soc. Mech. Eng. Ser. B 66 (2000) 679-685.

[48] J. V. Ramanamurthy, K. M. Prasad, V. K. Narla, Unsteady peristaltic transport in curved channels, Phys. Fluids 25 (2013) 091903(1-20).

[49] T. Hayat, A. Tanveer, F. Alsaadi, G. Mousa, Impact of radial magnetic field on peristalsis in curved channel with convective boundary conditions, J. Magn. Magn. Mater. 403 (2016) 47-59.

[50] N. Ali, K. Javid, M. Sajid, O. A. Bég, Numerical simulation of peristaltic flow of a biorheological fluid with shear-dependent viscosity in a curved channel, Comput Methods Biomech Biomed Engin 19(6) (2016) 614627.

[51] V. K. Narla, K. M. Prasad, J. V. Ramanamurthy, Peristaltic transport of jeffrey nanofluid in curved channels, Procedia Eng. 127 (2015) 869-876.

[52] V. K. Narla, K. M. Prasad, J. V. RamanaMurthy, Time-dependent peristaltic analysis in a curved conduit: Application to chyme movement through intestine, Math Biosci. 293 (2017) 21-28.

[53] A. A. Khan, FouziaMasood, R. Ellahi, M. M. Bhatti, Mass transport on chemicalized fourth-grade fluid propagating peristaltically through a curved channel with magnetic effects, J. Mol. Liq. 258 (2018) 186-195. 
[54] V. K. Narla, K. M. Prasad, J. V. RamanaMurthy, Second law analysis of a peristaltic flow of an incompressible viscous fluid in a curved channel, J. Eng. Phys. Thermophys. 89 (2016) 428-434.

[55] V. K. Narla, D. Tripathi, O. A. Bég, A. Kadir, Modelling transient magnetohydrodynamic peristaltic pumping of electroconductive viscoelastic fluids through a deformable curved channel, J Eng Math 111 (2018) 127-143.

[56] V. K. Narla, D. Tripathi, Electroosmosis modulated transient blood flow in curved microvessels: Study of a mathematical model, Microvasc Res 123 (2019) 25-34.

[57] A. M. Smith, A. A. Lee, S. Perkin, The electrostatic screening length in concentrated electrolytes increases with concentration, J. Phys. Chem. Lett. 7 (12) (2016) 2157-2163.

[58] S. M. S. Murshed, K. C. Leong, C. Yang, Thermophysical and electrokinetic properties of nanofluids - a critical review, Appl Therm Eng. 28 (2008) 2109-2125.

[59] R. C. D. Cruz, R. Jörg, O. M. S. Rainer, J. H. Michael, Electrical conductivity and stability of concentrated aqueous alumina suspensions, J. Colloid Interface Sci. 286(2) (2008) 579-588.

[60] J. Glory, M. Bonetti, M. Helezen, M. Mayne-L'Hermite, C. Reynaud, Thermal and electrical conductivities of water-based nanofluids prepared with long multiwalled carbon nanotubes, J. Appl. Phys 103(9) (2008) 094309 .

[61] A. Zayid, Factors Effecting the electrical conductivity and zeta potential of alumina nanofluids, Master's thesis, Dalhousie University Halifax, Nova Scotia, Canada, 2014. Master of Applied Science Dissertation. 